\title{
Discovering structural similarities among rāgas in Indian Art Music: a computational approach
}

\author{
H G RANJANI $^{1, *^{(D}}$, DEEPAK PARAMASHIVAN ${ }^{2}$ and THIPPUR V SREENIVAS ${ }^{1}$ \\ ${ }^{1}$ Department of Electrical Communication Engineering, Indian Institute of Science, Bangalore, India \\ ${ }^{2}$ Department of Music, University of Alberta, Edmonton, Canada \\ e-mail: ranjani@iisc.ac.in; paramash@ualberta.ca; tvsree@iisc.ac.in
}

MS received 16 August 2018; revised 4 February 2019; accepted 19 February 2019; published online 20 April 2019

\begin{abstract}
Indian Art Music has a huge variety of rāgas. The similarity across rāgas has traditionally been approached from various musicological viewpoints. This work aims at discovering structural similarities among renditions of rāgas using a data-driven approach. Starting from melodic contours, we obtain the descriptive note-level transcription of each rendition. Repetitive note patterns of variable and fixed lengths are derived using stochastic models. We propose a latent variable approach for raga distinction based on statistics of these patterns. The posterior probability of the latent variable is shown to capture similarities across raga renditions. We show that it is possible to visualize the similarities in a low-dimensional embedded space. Experiments show that it is possible to compare and contrast relations and distances between ragas in the embedded space with the musicological knowledge of the same for both Hindustani and Carnatic music forms. The proposed approach also shows robustness to duration of rendition.
\end{abstract}

Keywords. Indian Art Music; similar rāgas; rāga identification; repetitive note patterns.

\section{Introduction}

Rāgas, the melodic framework of Indian Art Music (IAM), are acknowledged to comprise grammatical structures [1]. The grammatical structures can be seen at three levels: (i) use of specific notes (swaras), (ii) ornamentations (gamakas) on the specific notes and (iii) note sub-sequences or phrases (prayōgas or sanchāras). The interaction amongst these creates a specific rāga-bhāva. The grammatical structures are said to result in varied groupings of rāgas based on number of notes, parent-derived $r \bar{a} g a$ or time of performance. These groupings arise from a musicological knowledge and perspective. In this paper, we intend to discover structural similarities among rāgas using a data-driven approach.

\subsection{Motivation}

In the musicological literature of IAM tradition (dominated by Hindustani and Carnatic music), rāgas form the melodic abstraction and contain grammatical structures.

Melody in IAM is defined through the rāga, which contains interactions of specific notes, their gamakas and the note transitions. The rules of such transitions are guided by well-defined grammatical structures of the $r \bar{a} g a$, which have been passed on from one generation to the other both

*For correspondence orally as well as aurally. In addition, the music form encourages extempore renditions and improvisations even while rendering a composition within the grammatical framework of a $r \bar{a} g a$. This results in melodic contour of any rendition not being deterministic and containing rāgaspecific nuances and improvisations, which themselves have a certain degree of variability within a given rāga.

Considering variabilities across rāgas from a musicological viewpoint, there have been various similarity attributes based on which rāgas have been grouped. Some of them are based on (i) number of notes (oudava, śâdava, mela classification), (ii) note movements (śuddha, chāyalaga or sankìrna classification [2]), (iii) rāga-rāgini classification [3], (iv) parent-derived classification (janaka-janya system in Carnatic and rāga-thāt system in Hindustani) [4, 5], (v) creative scope for development and the lack of it therein [6], (vi) time of performance (day/night/dusk rāgas) [4] and (vii) distinct emotional responses evoked as elucidated in the rāga-rasa theory [4]. Depending on the system of music, one or a few of these groupings are prevalent in the current performance practices of IAM. The basis of these groupings has been aurally taught or inferred by the connoisseurs.

In short, melodic similarities exist at various levels: within rendition, across renditions of the same rāga and across renditions of different rāgas. A rāga rendition will contain certain characteristic grammatical structures along 
with rendition-specific/composition-specific variabilities. A well trained listener can easily sieve grammatical structures from rendition-specific variabilities in order to compare and contrast its similarity to that of other renditions and rägas. In this work, we intend to discover the similarities between renditions based on melodic characteristics. We propose a data-driven computational approach to discover and analyse such similarity groupings. Such an analysis within and across $r \bar{a} g a$ renditions has applications in recommendation engines and cover song identification, and can potentially serve as an educational tool for music enthusiasts.

\subsection{Existing literature}

Data from all the three grammatical strata must be included to analyse structural similarities across rāgas. One way to ensure the inclusion of all the grammatical information is through the use of finely sampled pitch contours. Some approaches proposed in the recent years are [7-9] and [10]. In these works, rāgas with similar melodic characteristics are referred to as allied rägas or cohorts.

In the work proposed in [9], the authors extract finely sampled pitch contours from Carnatic dataset, pre-process it for tonic normalization and down-sample to $45 \mathrm{~Hz}$. Melodic similarity using dynamic time warping (DTW) is measured between sub-sequences of duration $2 \mathrm{~s}$ to discover patterns that are melodically similar. In [7], the same authors group similar melodic patterns and use vector space models based on tf-idf for rāga recognition in Carnatic music. Also, from the reported confusion matrices, the relations between allied rāgas can be observed.

The works in $[11,12]$ consider spotting and discovering typical motifs (defined as present in compositions of one raga and not in others) using stationary points of pitch contours. However, they do not address similarities/dissimilarities across ragas. In [8], the cohort of a rāga is defined as that $r \bar{a} g a$ with similar movements and subtle differences. The authors manually define cohorts of the considered 17 Carnatic rägas. The melodic contours are tonic-normalized pitch values in cent scale. Contours corresponding to pallavi section of the renditions are considered. Various contour matching algorithms such as DTW, Rough longest common sub-sequence (RLCS) and Longest Common Segment Set (LCSS) are used to obtain similarity scoring for $r \bar{a} g a$ verification and matching $[8,11,12]$.

In [10], the authors use pitch vectors within a 4-s window and use Locality Sensitive Hashing (LSH) algorithm to obtain top- $N$ matches for the queried rāga. The authors consider those rāgas that have similar melodic movements and common characteristic phrases but subtle differences as cohorts of a given rāga. Again, a list of cohorts are listed for each $r \bar{a} g a$ and the LSH matches are compared against a known cohort list for evaluation.

Summarizing, we observe that all of these methods propose to find repetitive sub-patterns within/across renditions followed by a method to group these sub-patterns and then look for similarity across the renditions. This is in accordance to the underlying role of repeated patterns and their lengths in characterizing similarities, which is well studied for music across various cultures [13, 14].

As observed from the work in [9], the large size of audio datasets, finely sampled melody contours coupled with absence of a reliable transcription system, grossly escalates the computations to be made to discover similarities across renditions and rāgas in IAM. We observe that mapping the pitch contours to a symbol set can potentially reduce the computational complexity of the problem at hand. This results in the challenges associated with retaining the finer nuances of ragas such as gamakas and note transitions.

\subsection{This work}

In this work, we propose an alternative framework of mapping the melodic contours to note sequences along with the gamaka information to discover and analyse similar rāgas. A note-sequence-based similarity analysis framework has some advantages over the pitch-contour-based analysis such as reduction of computational complexity, scope for inclusion of musicological knowledge as prior data and analysis of similarities (such as rāga similarity and composer similarities, which are relevant in the IAM context). However, considering the challenges in mapping melodic contours of IAM to note sequence $[15,16]$, this has not been a popular approach. The work in [17] proposed a method to address the challenge of automatically obtaining note sequences from melody contours by retaining much of the $r \bar{a} g a$ characteristics. We utilize this with the viewpoint that discrete entities are hypothesized to contain structural representations while expressions are realized in continuous variations as argued in [18].

In this paper, we consider the automatically obtained note level transcriptions, which can be extracted from melodic contours to aid in discovering structural similarities. We then estimate note sub-sequence statistics for each rendition. If the local temporal contexts are well captured in note sub-sequences, then the goal of similarity analysis across renditions through $r \bar{a} g a$ structure can be seen analogous to obtaining similarities across documents in natural language text processing (NLP). Hence, modelling rāgas in music can be seen as equivalent to topic modelling through the statistics of words and their contexts or utilizing temporal patterns and structures in genomic and/or protein sequences or capturing speaker/source characteristics through multiple speech utterances [19-25].

Starting from melodic contours of each rendition of a $r \bar{a} g a$, we obtain descriptive note-level transcriptions. Using these note sequences, we estimate repetitive note patterns (RNPs) and their probabilities (referred to as dictionary) for each rendition. The dictionaries of each rāga contain RNPs and their distributions. On the foundation of such 
constructed $r \bar{g} g a$ dictionaries, in this work, we (i) propose a feature representation for similarity analysis of rāgas, (ii) propose a model with a latent variable to represent rāgas, (iii) computationally discover similarities between rāgas through the structures captured by latent variable, (iv) analyse the effect of duration and other parameters on similarity of renditions and (v) use low-dimensional embedding techniques to visualize and measure similarities between renditions. The results obtained from the computational approach is compared and contrasted with three existing techniques inclusive of the state-of-art Time-Delayed-Melodic-Surface (TDMS) technique proposed in [26]. We further verify/validate the similarity results by seeking inputs from performing musicians of both Hindustani and Carnatic music forms.

In the following sections, we detail the proposed datadriven computational approach to discover the dominant similarity aspects across rāgas.

\section{Feature extraction}

We sample and quantize melodic contours of each rendition to obtain descriptive note-level transcriptions, which in turn form the features for further analysis. A block diagram representation of the same is shown in figure 1. The details of the same are as follows.

Tonic normalization is a standard approach and the first step used in analysis of multiple renditions of rāgas. The aim is to make the pitch contours invariant to different tonics (and hence to different artists/musicians). Let $y(t)$ be the melodic contour of a rāga rendition. Let the melodic contours of the datasets be tonic normalized and mapped to

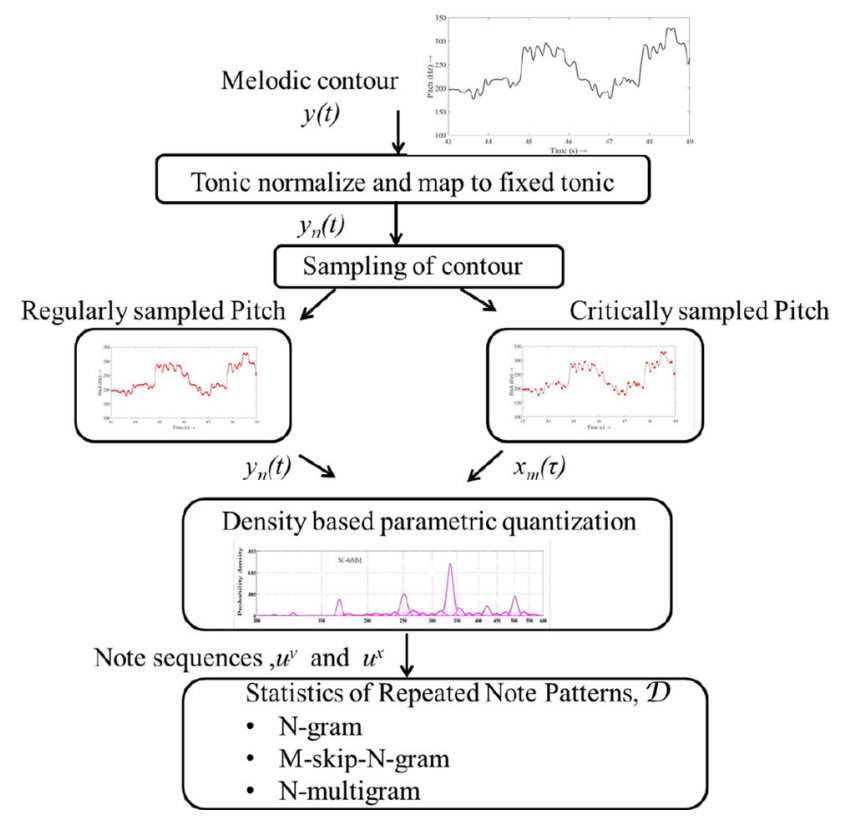

Figure 1. A block diagram depicting data flow for mapping of continuous melodic contour to discrete note sequences.
Table 1. Relative pitch positions, frequencies and ratio of notes within an octave.

\begin{tabular}{|c|c|c|}
\hline & Notes & $f_{0}$ in $\mathrm{Hz}$ (ratio with $S a$ ) \\
\hline 1 & $S a$ & $146.83(1)$ \\
\hline 2. & $R i_{1}$ & $156.66(16 / 15)$ \\
\hline 3. & $R i_{2}$ or $G a_{1}$ & $165.18(9 / 8)$ \\
\hline 4. & $R i_{3}$ or $G a_{2}$ & $176.19(6 / 5)$ \\
\hline 5. & $G a_{3}$ & $183.53(5 / 4)$ \\
\hline 6. & $M a_{1}$ & $195.28(4 / 3)$ \\
\hline 7. & $M a_{2}$ & $207.9(45 / 32)$ \\
\hline 8. & $\mathrm{~Pa}$ & $220.24(3 / 2)$ \\
\hline 9. & $D a_{1}$ & $234.92(8 / 5)$ \\
\hline 10. & $D a_{2}$ or $N i_{1}$ & $244.76(27 / 16)$ \\
\hline 11. & $D a_{3}$ or $N i_{2}$ & $264.29(9 / 5)$ \\
\hline 12. & $\mathrm{Ni}_{3}$ & $275.30(15 / 8)$ \\
\hline 13. & $\dot{S} a$ & $293.66(2)$ \\
\hline
\end{tabular}

a fixed tonic (chosen here as $f_{U}=146.83 \mathrm{~Hz}$ ) and be denoted by $y_{n}(t) \triangleq\left(y(t) / f_{T}\right) \times f_{U}\left(f_{T}\right.$ is the tonic frequency of original rendition). Let $\tau=\left\{t \mid \nabla y_{n}(t)=0\right\}$ be the set of critical points and $\boldsymbol{x}=\left\{y_{n}(\tau)\right\}$ be the corresponding critical pitch values. We model the probability density function (pdf) of $y_{n}(t)$ and that of the critical pitch values, $\boldsymbol{x}$, using a Semi-Continuous Gaussian Mixture Model (SC-GMM) as proposed in [27]. The means of the model, $\mu$, are fixed to pitch ratios as detailed in table 1 and the weights and variance, $\underline{\alpha}$ and $\underline{\sigma}$, respectively, are estimated. The parameter $\mu$ is agnostic to any rāga information, and the estimated $\alpha_{i}$ and $\sigma_{i}$ captures variations present in the rāga rendition. It is possible to realize note sequences from $y_{n}(t)$ in two possible ways using these SC-GMM parameters ${ }^{1}$ as proposed in [17]:

- $u_{t}^{y}=\arg \max _{i=1: K} \alpha_{i}^{y} \mathcal{N}\left(y_{n}(t) ; \mu_{i}, \sigma_{i}^{y}\right)$ where we quantize every pitch sample of $y_{n}(t)$. We refer to this as the QRP (Quantize Regularly sampled Pitch estimates) technique.

- $u_{m}^{x}=\arg \max _{i=1: K} \alpha_{i}^{x} \mathcal{N}\left(x_{m} ; \mu_{i}, \sigma_{i}^{x}\right)$ with $\left\{x_{m}\right\}$ as the critical points of $y_{n}(t)$. This is referred to as the QCP (Quantize Critical Pitch estimates) technique.

In this work, both the sequences are collectively referred to as $u_{m}$ and discerned based on technique used to realize them - QRP or QCP. Table 2 summarizes the two quantization techniques.

Consider the octave-folded note sequences, $u_{m}$, where $u_{m} \in V$, and $V=\{1,2, \ldots, 12\}$ is indicative of the pitch positions $\quad\left\{S a, R i_{1}, R i_{2}, G a_{2}, G a_{3}, M a_{1}, M a_{2}, P a, D a_{1}, D a_{2}\right.$, $\left.N i_{2}, N i_{3}\right\}$ (see table 1 for pitch ratios of the pitch positions). We refer to $V$ as the vocabulary in this work.

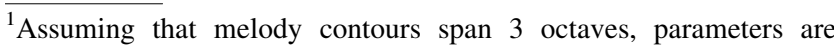
estimated using $K=12 \times 3$.
} 
Table 2. A brief over-view of quantization and sequencing schema proposed.

\begin{tabular}{|c|c|c|}
\hline Quantization schema & Overview & Parameters \\
\hline $\begin{array}{l}\text { Quantize regularly } \\
\text { sampled pitch } \\
\text { estimates (QRP) }\end{array}$ & $\begin{array}{l}\text { SC-GMM framework to } \\
\text { quantize pitch values } \\
\text { with pitch sampled at } \\
\text { regular time intervals }\end{array}$ & $\begin{array}{c}K \text { levels } F_{p^{-}} \\
\text {pitch } \\
\text { sampling } \\
\text { frequency }\end{array}$ \\
\hline $\begin{array}{l}\text { Quantize critical } \\
\text { pitch estimates } \\
(\mathrm{QCP})\end{array}$ & $\begin{array}{l}\text { SC-GMM framework to } \\
\text { quantize pitch values } \\
\text { with pitch sub-sampled } \\
\text { at critical points }\end{array}$ & $K$ levels \\
\hline Sequencing schema & Overview & Parameters \\
\hline$N$-gram & $\begin{array}{c}\text { Statistics of } N \text {-adjacent } \\
\text { notes }\end{array}$ & $N$ \\
\hline$M$-skip- $N$-gram & $\begin{array}{c}\text { Statistics of } N \text { notes } \\
\text { within a } N+M \\
\text { context }[28]\end{array}$ & $M$ skip, $N$ \\
\hline$N$-multigram [29] & $\begin{array}{c}\text { Statistics of } \leq N- \\
\text { adjacent notes }\end{array}$ & $N$ \\
\hline
\end{tabular}

Let $\boldsymbol{D}=\left[d_{1}, d_{2}, \ldots, d_{D}\right]$ represent a corpus of $D$ renditions. Let each rāga in $\boldsymbol{D}$ be represented by $J$ renditions and $R$ be the total number of rāgas considered; $D=J R$. We now model the note sequences of $u_{m}$ within each rendition. Let $s_{i}^{j}$ be the $i^{\text {th }}$ RNP (or note sub-sequence) estimated from $u_{m}$ note sequence of $j^{\text {th }}$ rendition. Let $\mathcal{D}^{(j)}$ be the dictionary for $j^{\text {th }}$ rendition, and is estimated from $u_{m}$ note sequences using 3 different stochastic models: $N$-gram, $M$-skip- $N$ gram and $N$-multigram models. The models consider both fixed length and variable length sub-sequences (or RNP). They are summarized in table 2. Thus, $\mathcal{D}^{(j)} \triangleq\left(s_{i}^{(j)}, \theta_{i}^{(j)}\right)$, where $i \in\left(1,2, \ldots,\left|\mathcal{D}^{(j)}\right|\right), j \in(1,2, \ldots, J R)$ and $\left|\mathcal{D}^{(j)}\right|$ is the number of elements of dictionary. The $s_{i}^{(j)}$ is the estimated RNP and $\theta_{i}^{(j)}$ is its probability of occurrence as obtained from the estimation techniques s.t. $\sum_{i=1}^{\left|\mathcal{D}^{(j)}\right|} \theta_{i}^{(j)}=1 \forall j$

Let $S \triangleq \cup_{r=1}^{R} \cup_{j=1}^{J} \cup_{i=1}^{\left|\mathcal{D}^{(j)}\right|} s_{i}^{(j)}$ be the collection of all RNP from the entire corpus. Then, for each technique, $S$ constitutes a bag of (fixed or variable length) sequences; this forms the common sub-sequence set for all the rāga renditions; $\theta_{i}^{(j)}$ which are in $S$, but not present in $\mathcal{D}^{(j)}$ are assigned zero (i.e., no smoothing is used). The union of RNP and the probability of their occurrences constitute a feature vector type of representation for further analysis. Let the total number of such RNP be $W=|\boldsymbol{S}|$. We create an RNP-Rendition (Rnp-R) matrix, $\boldsymbol{X}$, a $W \times D$ matrix (similar to term-document matrix in text processing), based on the probabilities of each rāga renditions; in other words, $x_{i j}=\theta_{i}^{(j)} \forall x_{i j} \in \boldsymbol{X}$. The two different quantization schemes, along with the three different stochastic models (summarized in table 2), are considered to obtain the RNP dictionaries, resulting in 6 types of Rnp-R matrix formulations.

\subsection{Datasets}

We use the publicly available CompMusic Hindustani and Carnatic rāga recognition datasets [7, 26]. The total duration of the dataset is about $120 \mathrm{~h}$ each. It contains audio recordings and the corresponding tonic information and pitch contours spanning 40 rāgas from Carnatic music and 30 rāgas from Hindustani music form. The pitch contour of each rendition has been extracted using Essentia toolkit [30]. The pitch contours are originally finely sampled at a rate of $4.4 \mathrm{~ms}$.

\section{Latent space rāga representation}

The variability across $\mathcal{D}^{(j)}$ for renditions of the same rāga could be due to the structure of the rāga and specificity of composition as well as due to the rendition style. The rendition dictionaries $\mathcal{D}^{(j)}$ are a possible representation of the specific rendition of the rāga. We hypothesize that the probability distribution of RNP captures the structural information about the rāga rendition. If this is a good representation for the rāga, then such a representation must result in the clustering of $r \bar{a} g a$-similar renditions (akin to semantic topic clustering of documents in natural text language processing). To explore the hypothesis, we formulate a generative model with a latent variable for the rendition-rāga-RNP process where rāga is the hidden latent variable. This latent variable represents a combination of RNP that best describes it. In effect, we hypothesize that a rāga represents a combination of dominant RNP. A graphical model of the same is depicted in figure 2. The model can be formulated as follows:

(i) choose a rendition, $d_{j}$;

(ii) let $P\left(r_{k} \mid d_{j}\right)$ be the probability of latent $r \bar{a} g a, r_{k}$, corresponding to $d_{j}$;

(iii) select RNP, $s_{i} \in \boldsymbol{S}$ with probability $P\left(s_{i} \mid r_{k}\right)$.

We have ignored the specific sequencing of the $s_{i}$ and assumed that $s_{i}$ are independent and can be obtained from the model. This is purely for modelling purposes and not for music generation/synthesis. For the $j^{\text {th }}$ rendition, $\theta_{i}^{(j)}$ are the probabilities, which form the $W$-dimensional observed feature vectors. Let $r \bar{a} g a$ be a latent variable such that RNP and rendition are conditionally independent given the latent variable. The asymmetric PLSA (probabilistic Latent Semantic Analysis), an unsupervised statistical clustering technique, is one such model that closely describes this data process [31], ${ }^{2}$ and expressed as

\footnotetext{
${ }^{2}$ For our application, we do not consider symmetric PLSA model where variables $d_{j}$ and $s_{i}$ are children of latent variable $r_{k}$. This would lead to a generative model where given latent $r \bar{a} g a, s_{i}$ and $d_{j}$ are sampled independent of each other. Such a model does not hold for the concerned application as there exist few $s_{i}$ that are unique to $d_{j}$, while others are common across renditions.
} 


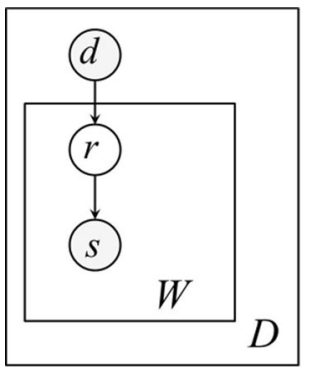

Figure 2. Graphical model depicting latent $r a \bar{g} a$ structure.

$$
P(\boldsymbol{S} \mid \boldsymbol{D})=\prod_{j=1}^{D} \prod_{i=1}^{W}\left(P\left(s_{i} \mid d_{j}\right)\right)^{c_{i j}}
$$

where $c_{i j}$ corresponds to the number of occurrences of the $i^{\text {th }}$ RNP in the $j^{\text {th }}$ rendition. $P\left(s_{i} \mid d_{j}\right)$ is modelled as follows:

$$
P\left(s_{i} \mid d_{j}\right)=\sum_{k=1}^{R} P\left(s_{i} \mid r_{k}\right) P\left(r_{k} \mid d_{j}\right) .
$$

If rāgas are akin to topics in NLP (natural language processing), $P\left(r_{k} \mid d_{j}\right)$ should result in clusters of renditions belonging to the same rāga, provided RNP is a good feature representation of the rāga. The model parameters $P\left(r_{k} \mid d_{j}\right)$ and $P\left(s_{i} \mid r_{k}\right)$ are estimated from the Rnp-R matrix using maximum likelihood (ML) approach, as detailed ahead.

\subsection{Inferring parameters from RNP-rendition matrix}

Given that renditions are independent, the complete likelihood can be written as

$$
\begin{aligned}
P(\boldsymbol{D}, \boldsymbol{R}, \boldsymbol{S}) & =\prod_{i=1}^{W} \prod_{k=1}^{R} \prod_{j=1}^{D}\left(P\left(s_{i} \mid r_{k}\right) P\left(r_{k} \mid d_{j}\right)\right)^{c_{i j}} \\
& =\prod_{i=1}^{W} \prod_{k=1}^{R} \prod_{j=1}^{D}\left(\phi_{i k} \pi_{k j}\right)^{c_{i j}}
\end{aligned}
$$

such that $\sum_{i=1}^{W} \phi_{i k}=1$ and $\sum_{k=1}^{R} \pi_{k j}=1$.

Let the posterior probability be $\gamma_{k i j} \triangleq P\left(r_{k} \mid s_{i}, d_{j}\right)$. Then, Expectation of Complete Log-Likelihood results in

$$
\begin{aligned}
Q= & \sum_{i=1}^{W} \sum_{k=1}^{R} \sum_{j=1}^{D} c_{i j} \gamma_{k i j}\left(\log \phi_{i k}+\log \pi_{k j}\right)+\lambda_{1}\left(1-\sum_{i=1}^{W} \phi_{i k}\right) \\
& +\lambda_{2}\left(1-\sum_{k=1}^{R} \pi_{k j}\right)
\end{aligned}
$$

ML estimation using Expectation Maximization (EM) algorithm (i.e., maximizing $Q$ w.r.t. $\phi$ and $\pi$ ) results in the following parameter update equations:

$$
\begin{gathered}
\phi_{i k}=\frac{\sum_{j=1}^{D} c_{i j} \gamma_{k i j}}{\sum_{j^{\prime}=1}^{D} \sum_{i^{\prime}=1}^{W} c_{i^{\prime} j^{\prime}} \gamma_{k i^{\prime} j^{\prime}}}, \quad \pi_{k j}=\frac{\sum_{i=1}^{W} c_{i j} \gamma_{k i j}}{\sum_{i^{\prime}=1}^{W} \sum_{k^{\prime}=1}^{R} c_{i^{\prime} j} \gamma_{k^{\prime} i^{\prime} j}} \\
\gamma_{k i j}=\frac{\phi_{i k} \pi_{k j}}{\sum_{k^{\prime}=1}^{R} \phi_{i k^{\prime}} \pi_{k^{\prime} j}} .
\end{gathered}
$$

We observe that the parameter estimation equations utilize the counts of $s_{i}$ in each rendition $d_{j}$, i.e., $c_{i j}$. We utilize probabilities obtained from dictionaries, $\theta_{i}^{(j)}$, in place of $c_{i j}$. This is possible as $\theta_{i}^{(j)}$ can be viewed as normalized counts, i.e., $\theta_{i}^{(j)}=\frac{c_{i j}}{\sum_{i=1}^{W} c_{i j}}$. These counts are obtained in different ways for $N$-grams, skip-grams and multigrams. With the introduction of $\theta_{i}^{(j)}$, the parameter update for $\pi_{k j}$ in (5) remains unchanged; however, the parameter $\phi_{i k}$ in (5) is now

$$
\phi_{i k}^{\prime}=\frac{\sum_{j=1}^{D} n_{j} c_{i j} \gamma_{k i j}}{\sum_{j=1}^{D} n_{j} \sum_{i=1}^{W} c_{i j} \gamma_{k i j}}
$$

where $n_{j} \triangleq \frac{1}{\sum_{i=1}^{W} c_{i j}}$. Then the posterior in (5) can be written as $\gamma_{k i j}^{\prime}=\frac{\phi_{i k}^{\prime} \pi_{k j}}{\sum_{k} \phi_{i k}^{\prime} \pi_{k j}}$. The advantage of using probabilities is that it ensures that contribution of all $s_{i}$ in every $d_{j}$ is proportionately weighed (see (1) and (2)), and irrespective of the total length of the rendition [32].

Since EM is used, the algorithm converges to a saddle point of the complete likelihood function. The algorithm is initialized with $\pi_{k j}$ to a uniform distribution and $\phi_{i k}^{\prime}$ to a (normalized) random vector. The posterior and parameter update equations are iterated till convergence. Here, convergence is defined as the point where rate of change of log-likelihood is less than a user-defined threshold (here set to 0.001$)$.

A sample evolution of $\pi_{k j}$ across iterations for samples of 2 rāgas (Māyamālavagowla and Sāvēri) from Carnatic dataset is shown in figure $3 ; \pi_{k j}$ can be observed to become sparse from a uniform initialization. The corresponding $\phi_{i k}$ obtained at convergence is shown with a word cloud representation of the RNP distribution in figure $4 .{ }^{3}$ For 1 -skip3-gram model, Hoyer's sparsity measure for $\pi$ is $\approx 0.6$ for both Carnatic and Hindustani datasets; similarly, sparsity measure for $\phi$ is found to be $\approx 0.8$ for both the datasets.

We reiterate that RNP distributions seen in the word clouds are ML estimates of the model for the given data. From the data-driven analysis, we observe that the two rāgas share some common RNP distributions $k=2,21,30$ while distinction arises due to the dominant presence of

${ }^{3}$ The usage of andholana gamaka in phrases such as sa ri sa ga ri sa in Māyamālavagowla or Sāvēri resulting in presence of latent $R i_{2}$ can be one possible musicological explanation for presence of $R i_{2}$ in word cloud. The musicological perspective to rāga similarities is addressed later in section 6.2. 
(a) Mayamalavagowla
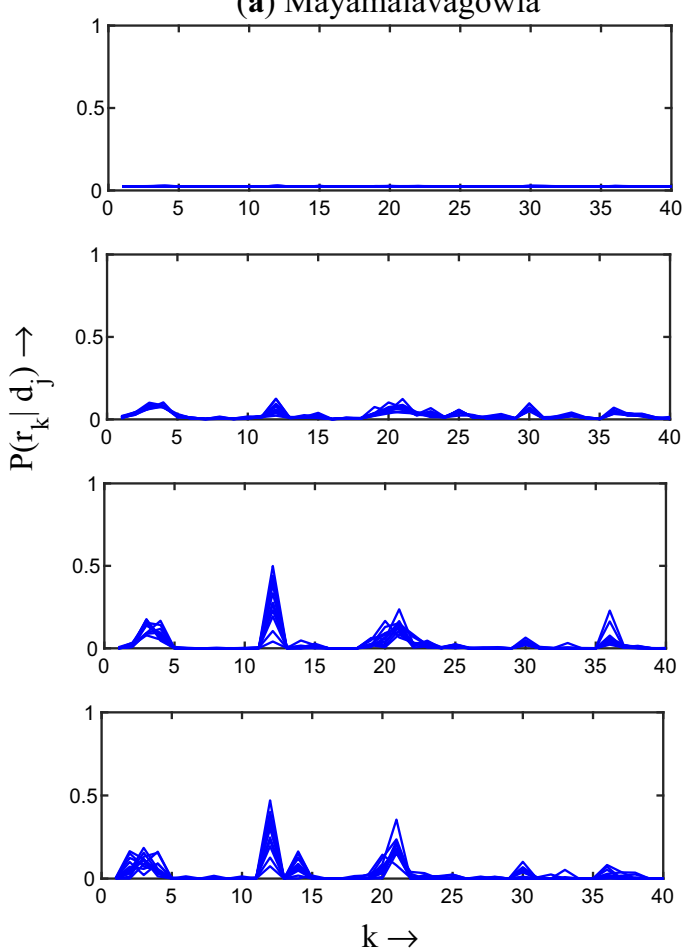

Iteration \#

2

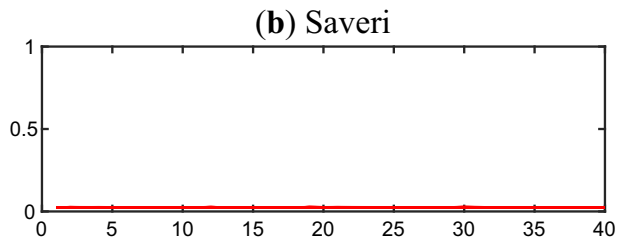

10

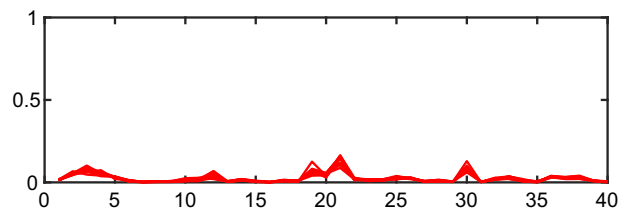

20

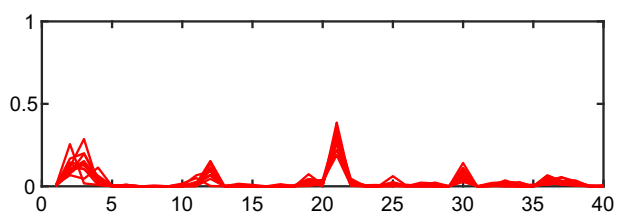

45

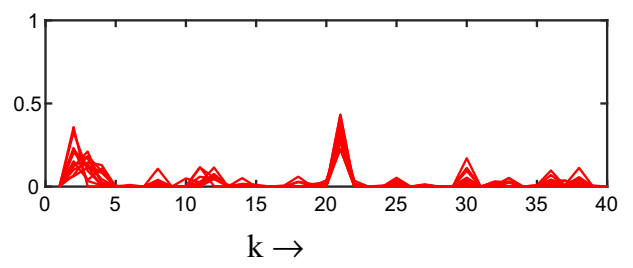

Figure 3. Evolution of $\pi_{k j}$ using 1-skip-3-gram model for 12 renditions each of two rāgas: (a) Māyamālavagowla and (b) Sāvēri of Carnatic music.
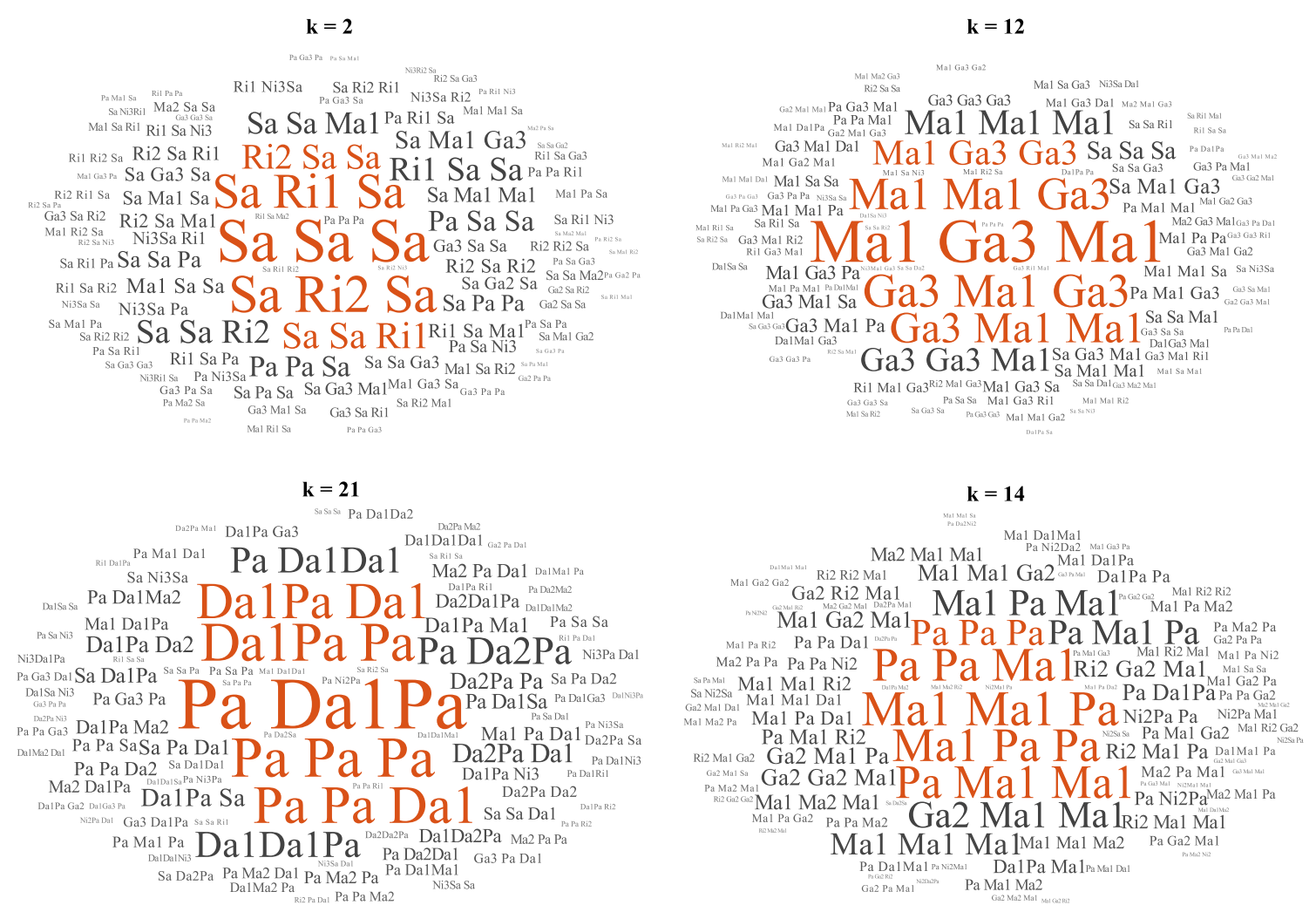

Figure 4. Word cloud representation for some selected $k$ depicting distribution of RNP as estimated from $\phi_{i k}$ using 1-skip-3-gram model for Carnatic dataset. 
some other RNP due to significance of distributions such as $k=12,14$ in Māyamālavagowla. An interested reader can find more examples of RNP distributions as word clouds for both Hindustani and Carnatic rāgas at https://tinyurl.com/ yad5utmk.

\subsection{Cluster purity}

Before venturing into supervised classification, we check if PLSA model can discover semantic structure of $r \bar{a} g a$ and hence result in $r \bar{a} g a$-specific grouping of renditions. We use cluster purity [33] of $P\left(r_{k} \mid d_{j}\right)$ to assess different methods of obtaining RNP. Cluster purity is defined as

$$
\mathcal{P}=\frac{1}{D} \sum_{m=1}^{C} \max _{\hat{k}}\left(a_{m, \hat{k}}\right)
$$

where $C$ is number of clusters and $a_{m, \hat{k}}$ is the number of renditions in cluster $m$, assigned to a class label $\hat{k}$. The class label is based on the identity of the rāga. A rendition can be assigned to a cluster label as $m=\arg \max _{k} \operatorname{Pr}\left(r_{k} \mid d_{j}\right)$.

Table 3 tabulates cluster purity (with $C=R$ ) for different combinations of quantization and sequencing schemes for both Hindustani and Carnatic music forms.

We see that QRP technique along with $N$-gram or $M$ skip- $N$-gram sequencing algorithm results in poor cluster purity for both Hindustani as well as Carnatic music forms (the same can also be visualized in figure 5(a)-(c)). The increase in length of RNP does not seem to help boost the clustering ability. By just changing the quantization scheme to QCP, the cluster purity almost doubles with unigram features. This shows that QCP is a better quantization scheme than QRP for analysing rāga clusters.

The cluster purity observed for Hindustani dataset is higher than that of Carnatic. This is because (i) number of raggas (classes) considered in Hindustani is 30 while that in Carnatic is 40 and (ii) Hindustani music contains more stable notes than Carnatic [34] and hence aids clustering.

These clusters are obtained with the QCP feature set. No explicit temporal information is used. It was observed that long stable notes were represented by multiple samples of same notes. We pre-process such stable notes and replace them with a representation of two repeated notes. As a result, the cluster purity of $M$-skip- $N$-gram is seen to increase for Hindustani music. This change in accuracy after the pre-processing results of the RNP distribution is less skewed by the sequences of single note, and could be more representative of distinctive RNP, thus helping cluster the rāgas better for Hindustani dataset. However, the same increase in cluster purity is not seen in case of Carnatic dataset. This can be due to the comparable contributions of transiting notes and stable notes in Carnatic music [35], and hence the pre-processing may not have a significant impact. For the Carnatic dataset, we observe that $M$-skip- $N$-grams for $M \neq 0$ result in better cluster purity than $N$-grams $(M=0)$ in most of the cases. This could be due to the

Table 3. Cluster purity for different quantization, sequencing techniques on Carnatic and Hindustani music dataset.

\begin{tabular}{|c|c|c|c|c|c|c|c|c|c|c|}
\hline \multirow{3}{*}{$\begin{array}{l}\text { Quantization } \\
\text { Technique }\end{array}$} & \multicolumn{2}{|c|}{ Sequencing } & \multicolumn{8}{|c|}{ Cluster purity } \\
\hline & \multirow[b]{2}{*}{ Algorithm } & \multirow[b]{2}{*}{ Parameter } & \multicolumn{4}{|c|}{ Hindustani } & \multicolumn{4}{|c|}{ Carnatic } \\
\hline & & & $M=0$ & $M=1$ & $M=2$ & $M=3$ & $M=0$ & $M=1$ & $M=2$ & $M=3$ \\
\hline \multirow[t]{5}{*}{$\mathrm{QRP}, K=12$} & $M$-skip- $N$ - & $N=1$ & 0.23 & & - & & 0.17 & & - & \\
\hline & gram & $N=2$ & 0.23 & 0.23 & 0.24 & 0.23 & 0.21 & 0.2 & 0.19 & 0.2 \\
\hline & & $N=3$ & 0.23 & 0.25 & 0.23 & 0.23 & 0.16 & 0.18 & 0.18 & 0.2 \\
\hline & & $N=4$ & 0.21 & 0.23 & 0.23 & 0.24 & 0.19 & 0.19 & 0.19 & 0.19 \\
\hline & & $N=5$ & 0.23 & 0.23 & 0.23 & 0.24 & 0.19 & 0.19 & 0.19 & 0.2 \\
\hline \multirow[t]{5}{*}{ QCP, $K=12$, } & $M$-skip- $N$ - & $N=1$ & 0.46 & & - & & 0.35 & & - & \\
\hline & gram & $N=2$ & 0.47 & 0.46 & 0.47 & 0.48 & 0.40 & 0.38 & 0.39 & 0.40 \\
\hline & & $N=3$ & 0.55 & 0.54 & 0.55 & 0.52 & 0.43 & 0.48 & 0.47 & 0.46 \\
\hline & & $N=4$ & 0.64 & 0.55 & 0.62 & 0.60 & 0.54 & 0.50 & 0.56 & 0.53 \\
\hline & & $N=5$ & 0.65 & 0.64 & 0.72 & 0.68 & 0.57 & 0.56 & 0.62 & 0.55 \\
\hline \multirow{5}{*}{$\begin{array}{c}\mathrm{QCP}, K=12, \\
\text { Pre-process }\end{array}$} & $M$-skip- $N$ - & $N=1$ & 0.44 & & - & & 0.32 & & - & \\
\hline & gram & $N=2$ & 0.49 & 0.47 & 0.47 & 0.49 & 0.39 & 0.36 & 0.40 & 0.39 \\
\hline & & $N=3$ & 0.69 & 0.63 & 0.63 & 0.62 & 0.46 & 0.48 & 0.50 & 0.46 \\
\hline & & $N=4$ & 0.70 & 0.76 & 0.67 & 0.66 & 0.50 & 0.53 & 0.51 & 0.58 \\
\hline & & $N=5$ & 0.63 & 0.74 & 0.66 & 0.69 & 0.52 & 0.56 & 0.59 & 0.57 \\
\hline \multirow{5}{*}{$\begin{array}{c}\mathrm{QCP}, K=12, \\
\text { Pre-process }\end{array}$} & $N$-multigram & & $N_{\min }=1$ & $N_{\text {min }}=2$ & $N_{\text {min }}=3$ & $N_{\text {min }}=4$ & $N_{\min }=1$ & $N_{\min }=2$ & $N_{\min }=3$ & $N_{\text {min }}=4$ \\
\hline & & $N=2$ & 0.46 & 0.51 & - & - & 0.41 & 0.44 & - & - \\
\hline & & $N=3$ & 0.56 & 0.64 & 0.63 & - & 0.49 & 0.48 & 0.49 & - \\
\hline & & $N=4$ & 0.56 & 0.64 & 0.63 & 0.60 & 0.46 & 0.45 & 0.43 & 0.52 \\
\hline & & $N=5$ & 0.34 & 0.41 & 0.38 & 0.41 & 0.31 & 0.33 & 0.30 & 0.34 \\
\hline
\end{tabular}




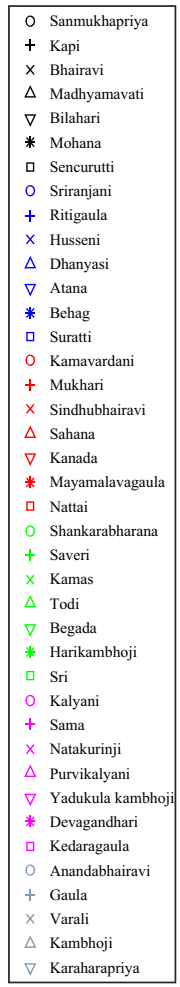

(I) Carnatic dataset
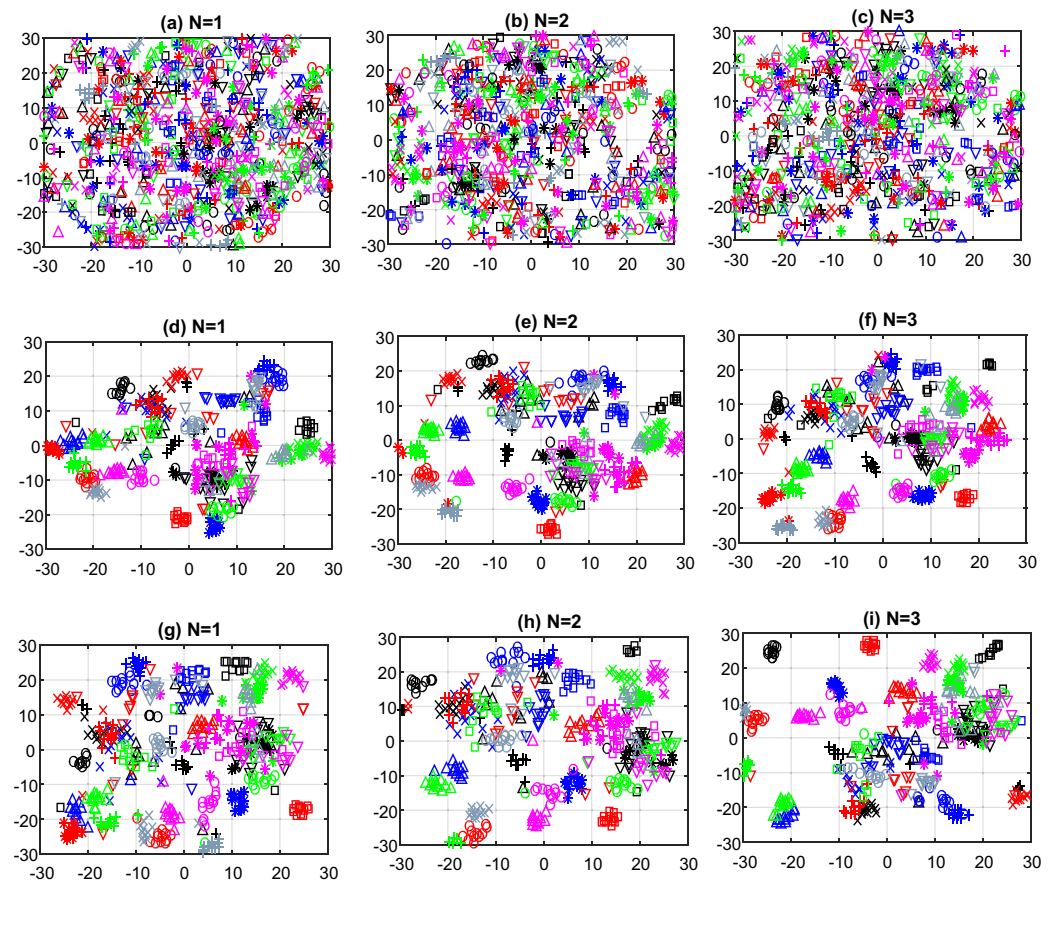

(II) Hindustani dataset
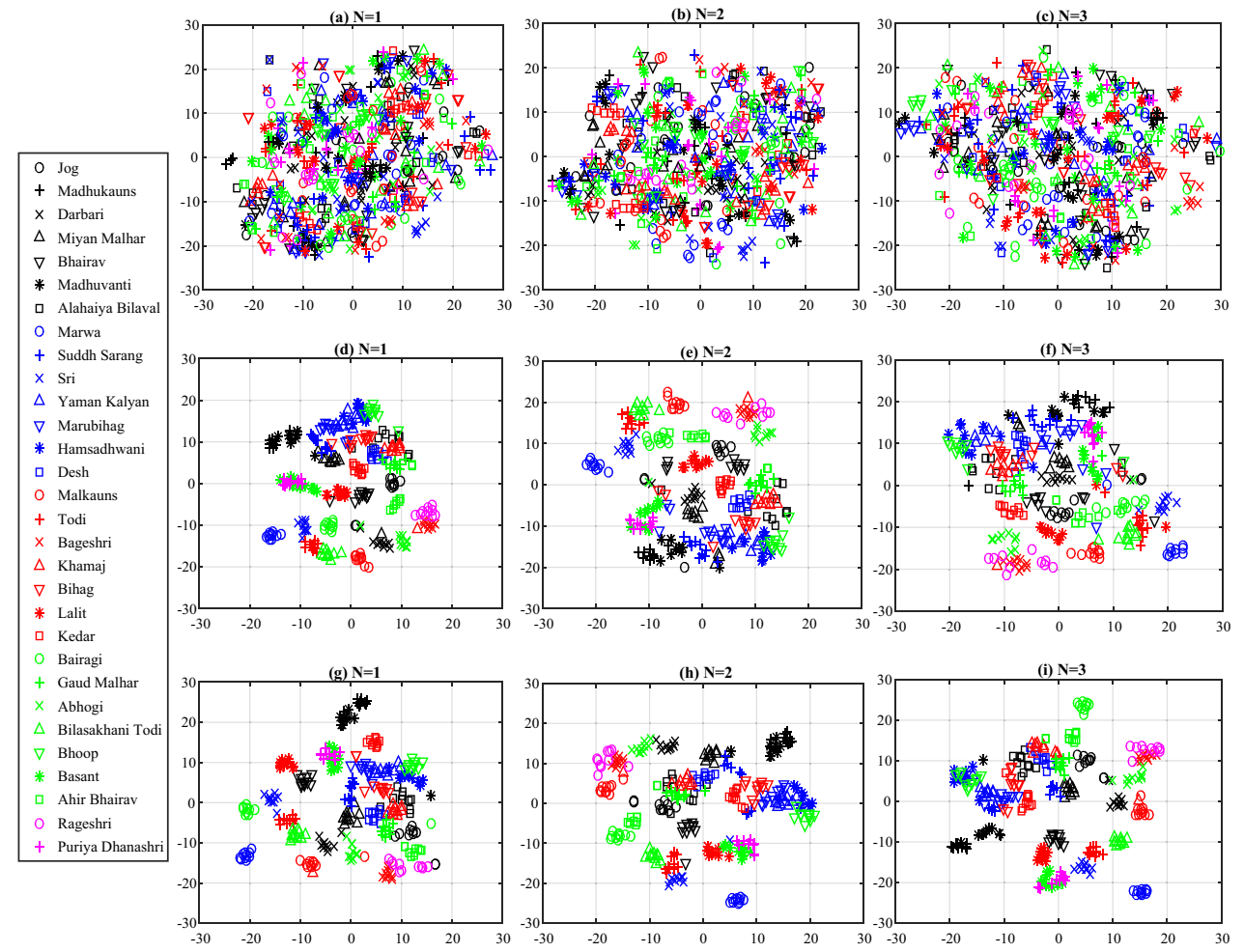

Figure 5. All plots are generated using $N$-gram sequencing technique to obtain $\boldsymbol{S}$. Plots (a), (b) and (c) are t-SNE plots (in 2-D) and use QRP quantization scheme along with PLSA for clustering. The subplots from (d) to (i) use QCP quantization scheme. The subplots from left to right correspond to 3 different $N$-gram sequences; $N=1(\mathbf{a}, \mathbf{d}, \mathbf{g}), N=2(\mathbf{b}, \mathbf{e}, \mathbf{h}) N=3(\mathbf{c}, \mathbf{f}, \mathbf{i})$. The top row $(\mathbf{a}, \mathbf{b}, \mathbf{c})$ and bottom row $(\mathbf{h}, \mathbf{i}, \mathbf{j})$ correspond to dimension-reduced t-SNE plots (in 2-D) obtained from $P\left(r_{k} \mid j\right)$ terms of PLSA while the plots of the middle row $(\mathbf{d}, \mathbf{e}, \mathbf{f})$ correspond to t-SNE clusters obtained from $\boldsymbol{X}$. 
strength of skip-gram models to account for variations (such as sangatis within a rendition).

The cluster purity using the $N$-multigram model is much lower than $M$-skip- $N$-grams for both Hindustani and Carnatic music. This implies that RNP obtained from $N$ multigram model may not result in distinctive clusters of rāgas. This is because the distributions estimated using ML criterion may be sub-optimal compared with the distributions obtained by sequence counts (as in $N$-gram). It also implies that considering some of the notes in a sub-sequence of note may be advantageous with the descriptive transcripts. This is in contrast with the observations that can be made from study of data from prescriptive notations [36]. This is explainable as follows: gamaka variations in a rāga are captured in successive notes in descriptive transcripts, whereas the same are not considered/notated in prescriptive notations. The successive notes can/will include variations specific to singer/composition in a descriptive transcription while the same will not be included in a skeletal, prescriptive version.

\subsection{Analysis}

The possibility of clustering renditions based on rāgas using basic $N$-gram is shown in figure 5 for $N=1,2$ and 3 for Hindustani and Carnatic datasets. It depicts low dimension embeddings obtained using t-SNE algorithm (t-distributed Stochastic Neighbour Embedding) [37]. It can be observed from figure 5(a) that a direct dimensionality reduction with distributions $\boldsymbol{X}$, obtained using QRP, does not result in clusters. From figure 5(b) and (c), we see that increasing $N$ does not aid in clustering. This visualization corroborates the results observed with cluster purity measures. From figure 5(d)-(f), we see the presence of clusters with QCP quantization technique. However, the cluster separability is not seen with increasing contexts. With introduction of a latent variable $r_{k}$, renditions tend to form well-separated rāga-specific groups (see figure 5(g)-(i)). We infer, from cluster purity table and visualization using t-SNE, that mere distribution of notes (unigram or $N=1$ ) may not result in $r \bar{g} g a$-specific grouping of renditions and the increase of parameter $N$ (length of RNP or sub-sequences with more context) results in more compact clusters. Further, introducing latent variable $r_{k}$ results in well-separated clusters. These observations hold with respect to both the datasets.

We study the effect of number of clusters and quantization levels on rāga-specific grouping of renditions for the proposed latent variable model.

\subsection{Effect of parameters}

We had considered the case of $C=R$ and analysed for cluster purity till this point. The effect of number of clusters, $C$, and that of the quantization depth, $K$, is explored here. 3.4a Number of clusters, C: In order to estimate the quality of $r \bar{g} g a$ representation with changing number of clusters, we consider the Akaike Information Criterion (AIC) [38], which is defined as $A I C \triangleq 2 C-2 \log (L)$ where $2 C$ represents the penalty term for increasing number of clusters and $L$ is likelihood. Figure 6 depicts the change in $A I C$ as a function of $C$. We see that $A I C$ is minimum at $C \simeq R$ for both Hindustani and Carnatic datasets. This in turn reinforces that the latent variable $r_{k}$ in turn truly models the rāga.

We can visualize the effect of $C$ on clustering for Hindustani and Carnatic datasets with figure 8 under 3 conditions:

- $C<R$. The number of latent variables and (hence clusters) is less than required. The clusters contain more diverse renditions.

- $C \simeq R$. Visually, we observe that the clusters tend towards containing rāga-specific renditions.

- $C>R$. The clusters become wide spread possibly due to other variabilities such as lyrics and singer style.

The clusters are observed to be compact when $C \simeq R$.

3.4b Effect of quantization depth and vocabulary size: It is possible that the depth, $K$, of the quantization techniques or the vocabulary size $V$ has an impact on the clusters formed. The cluster purity evaluations of table 3 corresponds to $K=12 \times 3$ quantization levels and octave-folded vocabulary with $|V|=12$. We now consider 4 different vocabulary sizes, which in turn correspond to the number of distinct notes identified in Indian musicology.

- $|V|=7$ case. This is motivated from the solfège equivalent in IAM, which considers 7 syllables [6]. $K=12 \times 3$ quantization levels and its octave-folded note sequence is considered. The $|V|=12$ vocabulary

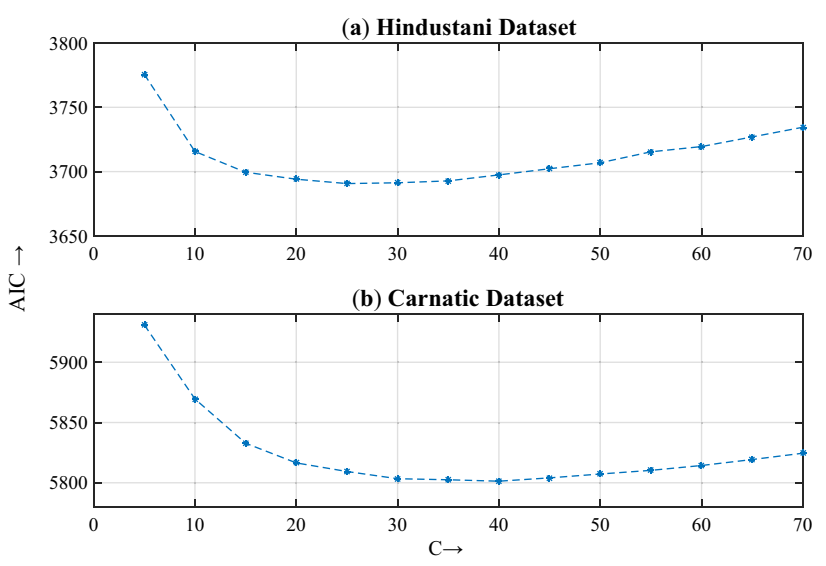

Figure 6. $\mathrm{AIC}$ as a function of number of clusters $C$ for (a) Hindustani dataset with $R=30$ and (b) Carnatic dataset with $R=40$. 
(a) Carnatic

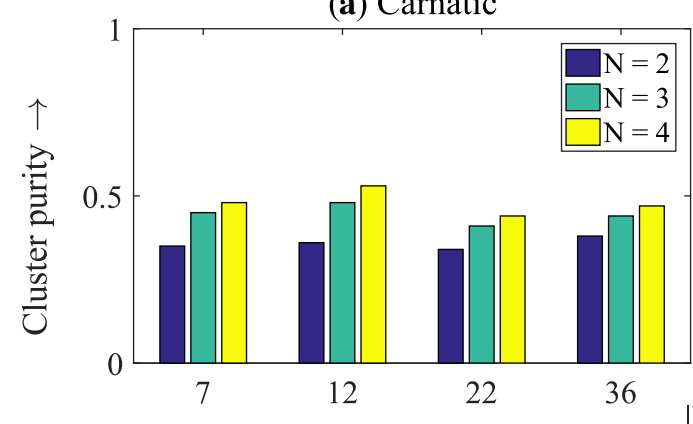

(b) Hindustani

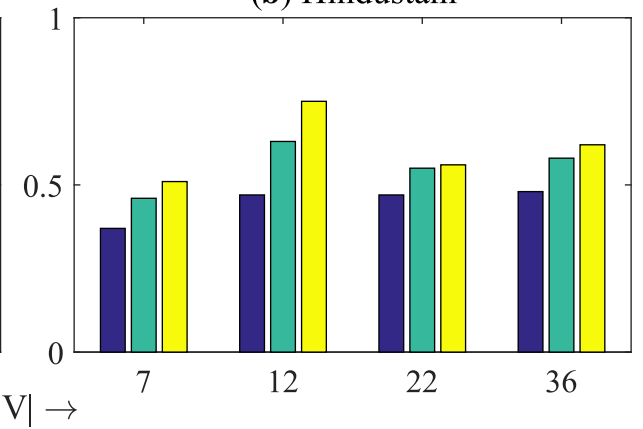

Figure 7. Effect of $|V|$ on cluster purity of $M$-skip- $N$-gram (with $M=1$ and $N=3$ ) for (a) Carnatic and (b) Hindustani datasets.

is further folded to ignore the finer note positions, resulting in $|V|=7$.

- $|V|=12$ case. The 12 notes within an octave system are the most prevalent. We consider 3 octaves, and hence $K=12 \times 3$ quantization levels are considered, followed by folding the notes across octaves to obtain $|V|=12$.

- $|V|=22$ case. This is motivated from the micro-tonal intervals that have been suggested for IAM [6]. Though this is controversial, we consider this for objective analysis of the data. The $K=22 \times 3$ quantization levels across 3 octaves are folded to obtain $|V|=22$.

- $|V|=36$ case. Here, we consider $K=12 \times 3$ quantization levels, which forms the vocabulary.

Figure 7 shows the cluster purity for different $|V|$ using the $M$-skip- $N$-gram model with $M=1, N=3$.

With increase in the number of quantization levels and the vocabulary size, i.e., $|V|=22$ or $|V|=36$, it is observed that cluster purity reduces for all of the sequencing schemes. This is observed for both Hindustani and Carnatic datasets. On increasing the number of quantization levels, we are increasing the variety of possible sub-sequences. Similar patterns are not captured effectively with $|V|=22$ as the distribution so obtained is more spread than with $|V|=12$. In case of $|V|=36$, octave errors present in the melodic contour can alter the distribution of sequences. Analysing the cluster purity for $|V|=7$, the reduction in cluster purity is mainly seen due to RNP being clubbed together-this does not result in distinctive clusters for each rāga.

\section{Distances between rāga renditions}

Any change in number of clusters, quantization depth and vocabulary size is seen to have an adverse effect on clustering of rāgas (figure 8). For the rest of this manuscript, we consider the optimum parameter setting of $C=R$ and $|V|=12$ unless specified otherwise.

(I) Hindustani dataset
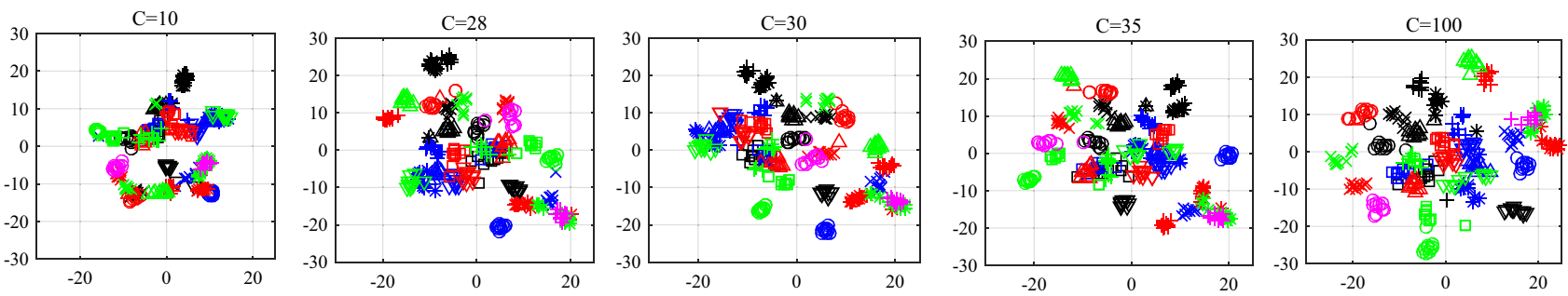

(II) Carnatic dataset
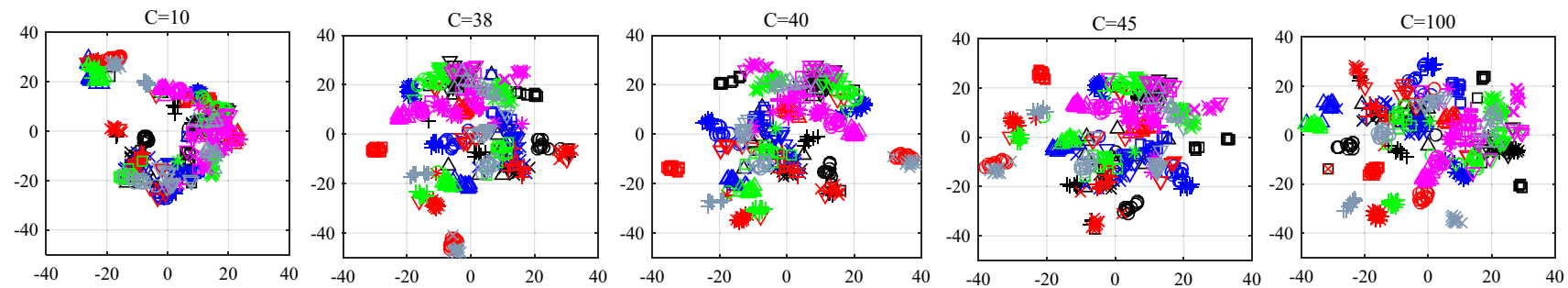

Figure 8. t-SNE plots of clusters depicting $P\left(r_{k} \mid d_{j}\right)$ for various $C$. (I) Hindustani dataset with $R=30$. (II) Carnatic dataset with $R=40$. 
With the rāga clusters appearing well arranged, it is possible to hypothesize a space for the rāga renditions. This helps in comparing/contrasting renditions from the musicological perspective using distance measures. We formulate 3 ways to address distance measures between renditions: (i) directly from RNP dictionaries, (ii) using PLSA-decomposed latent variable vector space and (iii) using a lower-dimensional embedding space. We consider the first two approaches in this section and later consider lower-dimensional embedding space in section 6 .

\subsection{Using RNP dictionaries}

We have seen that the renditions do not necessarily form clusters as per the underlying rāga if RNP dictionaries are directly used (see figure 5(d)-(f)). We can quantify the same using the distances between renditions. The distances can be compared by constructing similarity matrices. The symmetric KL-Divergence is proposed as a distance metric between RNP dictionaries of two renditions, i.e.

$$
\begin{aligned}
\operatorname{DIST}\left(d_{j}, d_{l}\right)^{r n p}= & \sum_{i} P\left(s_{i} \mid d_{j}\right) \log \frac{P\left(s_{i} \mid d_{j}\right)}{P\left(s_{i} \mid d_{l}\right)} \\
& +\sum_{i} P\left(s_{i} \mid d_{l}\right) \log \frac{P\left(s_{i} \mid d_{l}\right)}{P\left(s_{i} \mid d_{j}\right)} .
\end{aligned}
$$

\subsection{Using PLSA-decomposed latent variable space}

If a rāga can be represented by a convex combination of dominant RNP, then the combinations used across renditions of a rāga must be similar. Hence, the distances between $P\left(r_{k} \mid d_{j}\right)$ should also reflect the same. For the PLSA formulation of rāga model, the distance between $P\left(r_{k} \mid d_{j}\right)$ and $P\left(r_{k} \mid d_{l}\right)$ is proposed as distance measure between $d_{j}$ and $d_{l}$ renditions $\forall j, l \in[1, D]$. We use symmetric KLDivergence as the distance metric, i.e.

$$
\begin{aligned}
\operatorname{DIST}\left(d_{j}, d_{l}\right)^{p l s a}= & \sum_{k} P\left(r_{k} \mid d_{j}\right) \log \frac{P\left(r_{k} \mid d_{j}\right)}{P\left(r_{k} \mid d_{l}\right)}+ \\
& \sum_{k} P\left(r_{k} \mid d_{l}\right) \log \frac{P\left(r_{k} \mid d_{l}\right)}{P\left(r_{k} \mid d_{j}\right)} .
\end{aligned}
$$

Figure 9 depicts similarity matrix for a sub-set of Carnatic dataset. The similarity matrix is expected to show block diagonal structures indicative of similarity across renditions of the same rāga. We observe more pronounced block diagonal structures through the use of DIST ${ }^{\text {plsa }}$ than with similarities obtained using $D I S T^{r n p}$. To validate similarities observed with the proposed approach, we evaluate rāga identification accuracy.

\section{Rāga identification}

In this section, we report rāga identification accuracy for both datasets. The leave-one-out cross-validation framework is used and $K$-nearest neighbor algorithm is considered for identification task. We consider two distance metrics for the identification task:

- DIST ${ }^{r n p}$ enables evaluation of direct use of RNP for rāga identification task.

- DIST ${ }^{p l s a}$ evaluates the PLSA-based latent variable approach. We first estimate $P\left(s_{i} \mid r_{k}\right)$ and $P\left(r_{k} \mid d_{\text {train }}\right)$ in the PLSA training phase using all the renditions except the test rendition. For the test data, $P\left(s_{i} \mid r_{k}\right)$ as estimated from training phase is fixed and only $P\left(r_{k} \mid d_{\text {test }}\right)$ is estimated. For each $P\left(r_{k} \mid d_{\text {test }}\right)$, the $r \bar{g} g a$ label corresponding to that of the nearest neighbour, with $D I S T^{p l s a}\left(d_{j}, d_{t e s t}\right)$ metric, is used.

The recall percent for using both the techniques is shown in figure 10 . We see that the recall percent of the identification task is always low for the case of DIST ${ }^{r n p}$. This further reflects that the RNP do not directly aid in rāga identification task. For further evaluation with existing methods, we compare PLSA-based latent variable approach.

We consider three different existing methods in the literature: (i) TDMS (Time Delayed Melodic Surface proposed in [26]), (ii) PCD (Pitch Class Distribution proposed in [39]) and (iii) VSM (Vector Space Model proposed in [7]). To ensure a fair comparison, performance accuracy reported for all algorithms uses the leave-one-out framework. We compare the performance of PLSA-based $N$ gram, $M$-skip- $N$-gram-based and $N$-multigram-based approaches with the performance of the existing methods. The recall percentage for various methods is depicted in figure 11 .

We see that TDMS algorithm shows superior performance for the task. An improved performance with 100 cents per bin (without compression and smoothing) was seen than the originally proposed 10 cents per bin. This modified version without smoothing and compression as proposed by the authors shows the highest rāga identification accuracy for both the datasets. We see that the PLSA-based methods outperform the VSM technique for both Carnatic and Hindustani datasets. Further, the proposed QCP-based RNP dictionaries followed by PLSA clustering approach outperform PCD technique for Carnatic dataset.

Observing the performance of QCP techniques on rāga identification task, we see that skip-grams with $N=3$ perform well and the performance degrades with increasing skip $(M)$ and $N$. This is because the matrix $\boldsymbol{X}$ becomes sparser and longer sequences may not be frequently available in every rendition, thus reducing the count $c_{i j}$ for a subsequence $s_{i}$. We can correlate this observation with 
(a) $\operatorname{DIST}^{\mathrm{rnp}}$

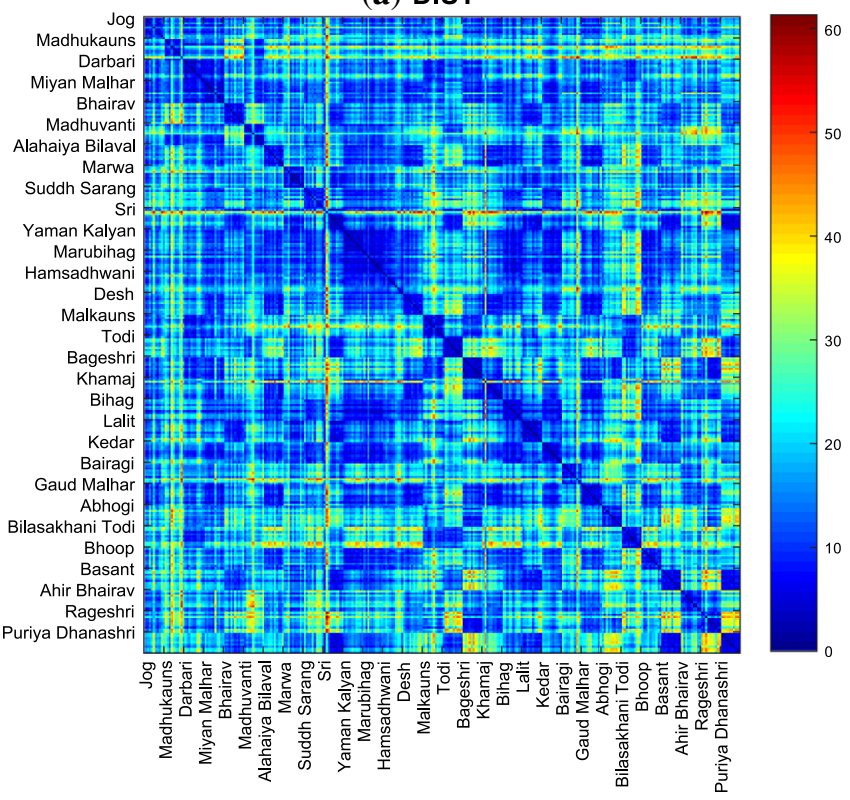

(c) DIST ${ }^{\mathrm{rnp}}$

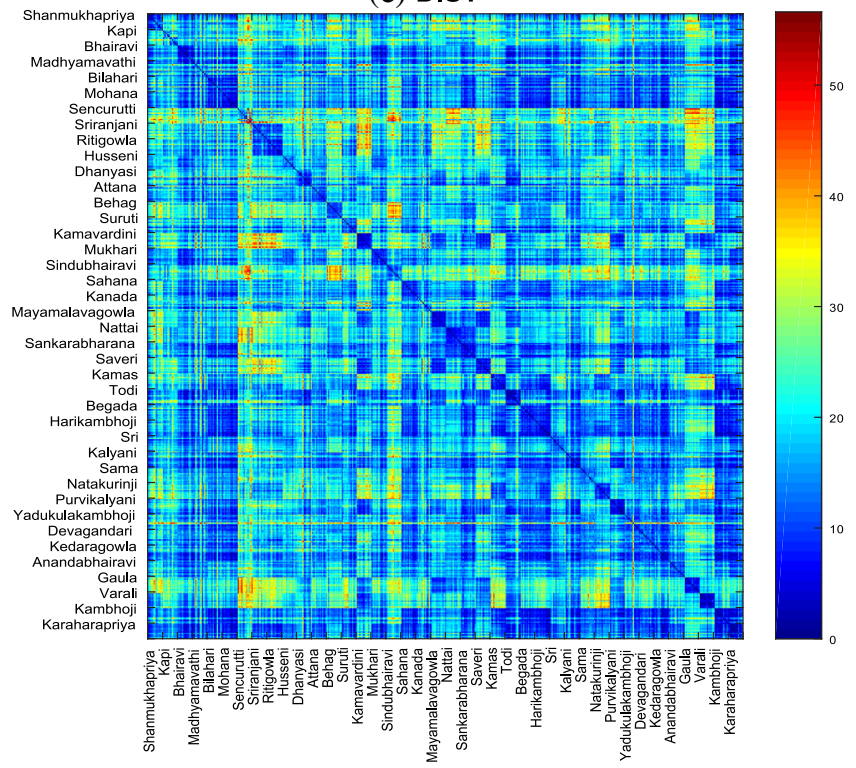

(b) DIST $^{\text {plsa }}$

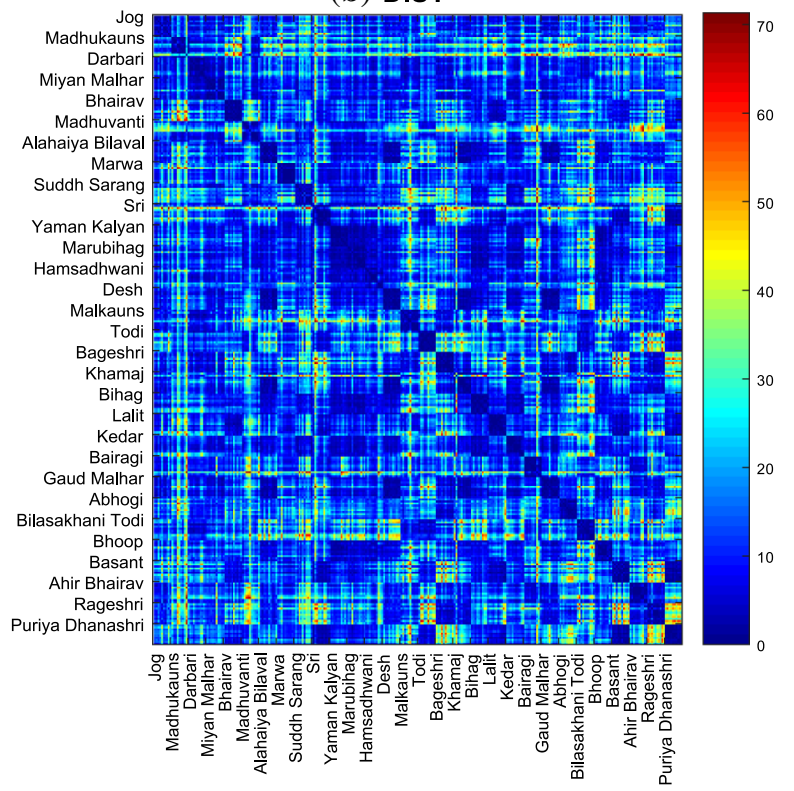

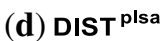

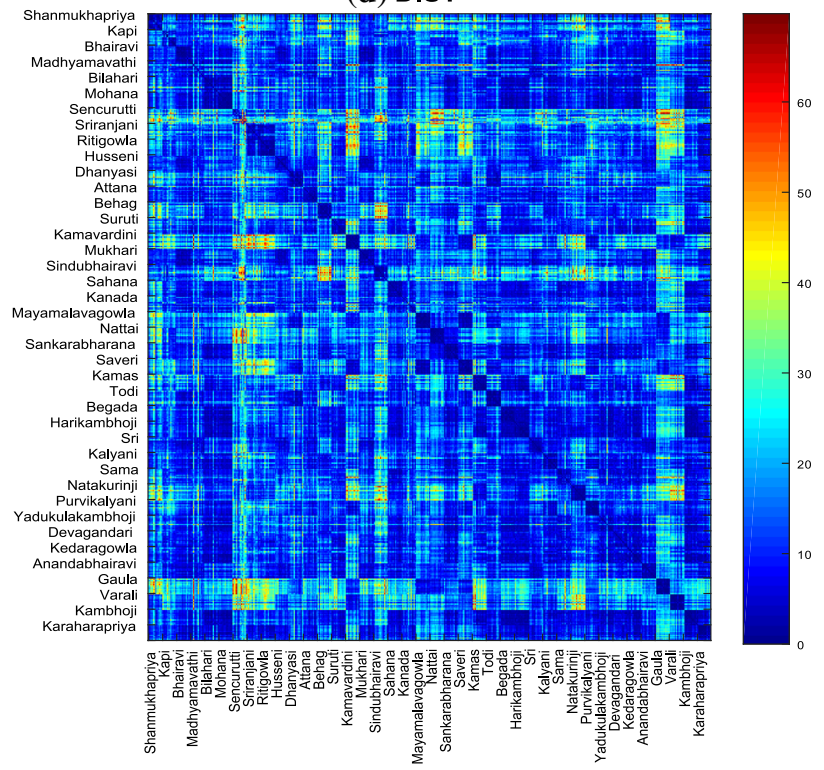

Figure 9. Similarity matrix for Hindustani dataset $(\mathbf{a}, \mathbf{b})$ and Carnatic dataset $(\mathbf{c}, \mathbf{d})$ showing the block diagonal structure obtained using $D I S T^{r n p}$ and $D I S T^{p l s a}$.

languages where tri-grams are popular; estimating parameters of higher grams require more training data [40].

The rāga identification accuracy obtained using the distances between renditions in the t-SNE-embedded space is observed to closely follow that reported using PLSA approach. This implies that distances between renditions can be measured even from the embedded space.

We have considered the entire rendition for $r \bar{g} g a$ analysis till this point. We now study the effect of reduced duration of renditions on rāga identification.

\subsection{Effect of duration of test data on rāga identification}

Identifying a rāga within minimum exposition of a rendition is a primary and important aspect of any IAM concert [12]. With all performers and connoisseurs aiming towards this goal, it is natural that a rāga identification system must address this aspect of a rendition. Hence, we analyse the effect of reducing the duration of test renditions on the performance of rāga identification task. We consider two scenarios: (i) the rendition duration is halved, i.e., only first 


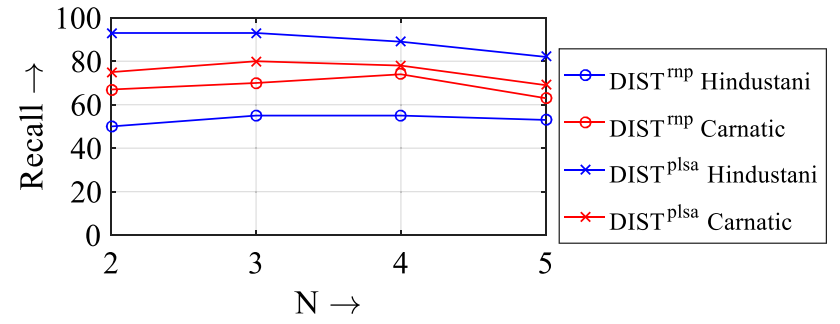

Figure 10. Rāga identification accuracy using $M$-skip- $N$-gram ( $M$ fixed to one) dictionaries and DIST ${ }^{r n p}$ and DIST ${ }^{p l s a}$ as distance metric for K-Nearest Neighbor classifier.

half is retained and (ii) only the first minute of rendition is retained. The change in accuracy of rāga identification using different algorithms is plotted in figure 12 for Carnatic and Hindustani datasets.
From figure 12, it can be observed that when the duration of test rendition is halved, the recall accuracy does not change significantly in any of the algorithms. However, when only the first minute of rendition is tested, the recognition accuracy reduces to $\sim 60 \%$ for Hindustani dataset. For Carnatic dataset, the fall in recall accuracy of TDMS technique is large (to $\sim 32 \%$ ); the skip-gram techniques show more robustness to less data in both Carnatic and Hindustani music. Further, amongst the QCP techniques, the reduction of accuracy in tri-gram is much more than in skip-grams. This result highlights that there are improvisations present even in first minute of the data, which contribute greatly to the statistics of sequences. It can also be observed that the rāga identification accuracy drops more for the Hindustani dataset than that of Carnatic.

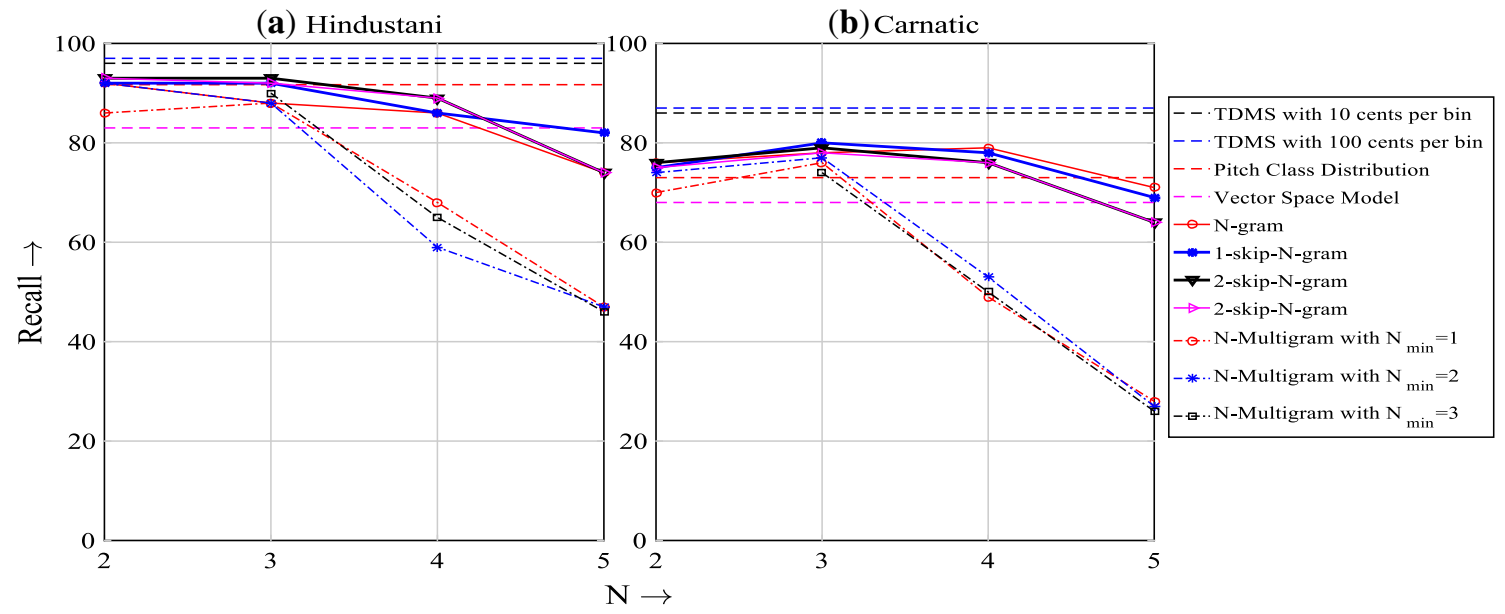

Figure 11. Rāga identification accuracy for Carnatic and Hindustani datasets using different algorithms.

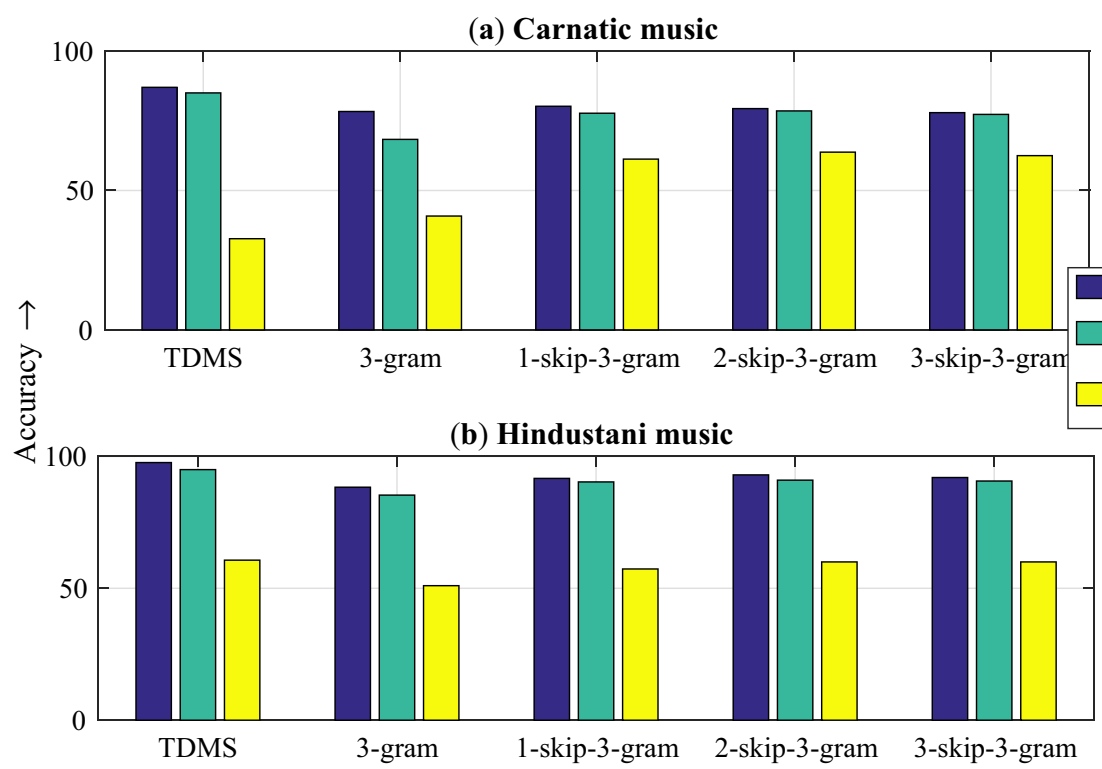

Figure 12. Rāga identification recall accuracy for different durations of data from (a) Carnatic dataset and (b) Hindustani dataset. 
(a) Full length testing
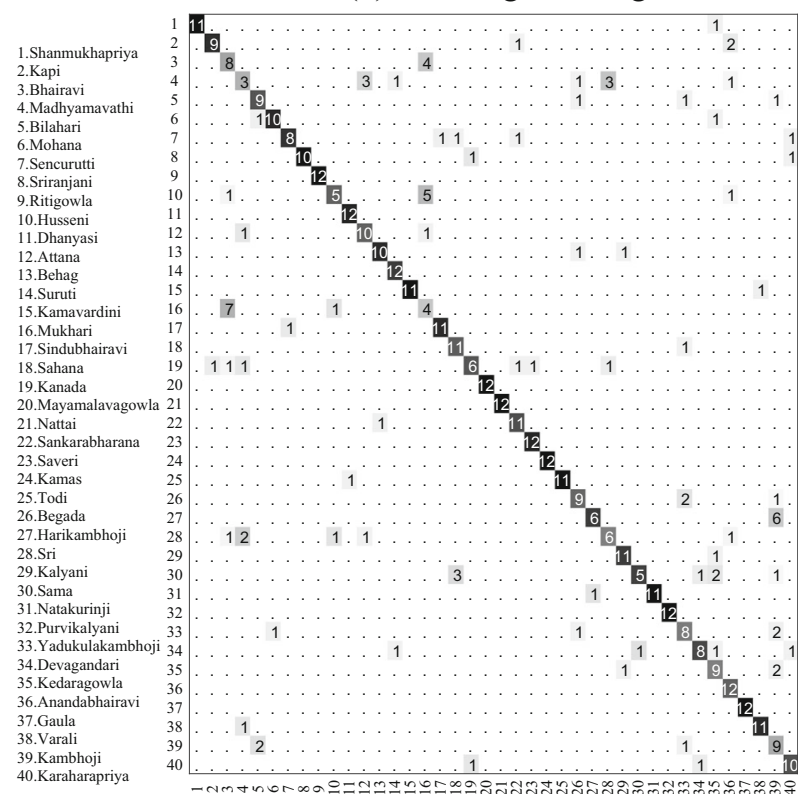

(b) First minute testing

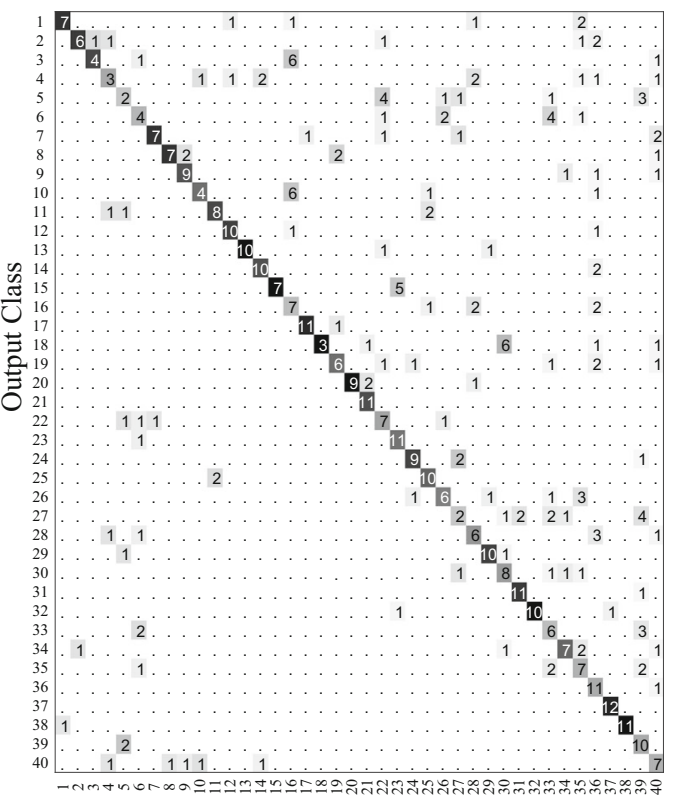

Target Class

(c) Full length testing

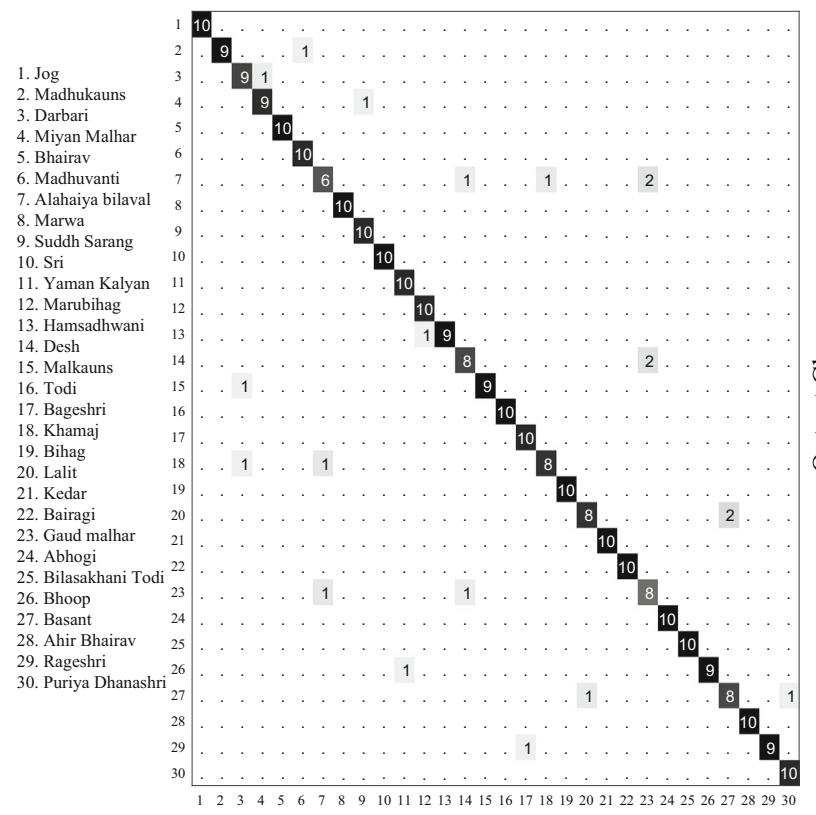

(d) First minute testing

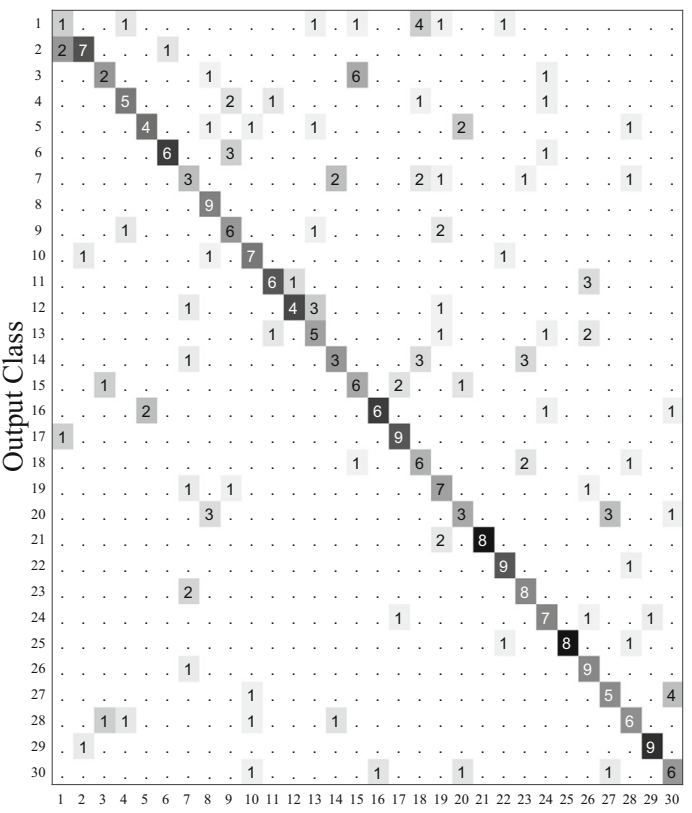

Target Class

Figure 13. Confusion matrices for (a,b) Carnatic and (c,d) Hindustani music using 2-skip-3-gram sequence statistics.

This is perhaps due to the fact that in Hindustani music, the artist may not have depicted all the characteristics of the rāga in the first minute of the rendition.

Figure 13 shows confusion matrices for both Hindustani and Carnatic using 2-skip-3-gram algorithm for test data comprising (i) entire duration of the rendition and (ii) only the first minute of rendition. When we analyse the confusion matrix for Carnatic music, we see that considerable samples of Bhairavi and Hus̄eni are consistently confused as Mukhāri, Sahana as Sama, HariKāmbhoji and Yadukula-Kāmbhōji as Kambhoji, Bēgada as Kēdāragowla, Bilahari as Shankarābharana and Kāmavardhini as Sāvēri. Musicologically, these rāgas are based on same notes and it is reasonable to expect some 
similarity. Though the characteristic phrases of the rāga would have been introduced early in the rendition, it is possible that the test data are insufficient for correct classification. Contrarily, it is interesting to note that some Mukhäri pieces that were misclassified as Bhairavi when full test data were available were not mis-classified when only one-minute data were considered. This implies that the initial expository portion of rendition contains characteristics of a rāga and is in alignment with the findings in [10].

Similarly, analysing the confusion matrices for Hindustani music on first minute of rendition, we see that Darbari is confused as Malkauns, Jog and Dēsh as Khamaj, Madhuvanti as Shuddh Sārang, Lalit as Basant, Basant as Puriya Dhanashrē and Yaman Kalyān as Bhoop. These rāgas are musicologically similar. This is expected in Hindustani music as the characteristic phrases of the rāga would have been introduced much later in the rendition. This shows that duration of test data is more critical for Hindustani music.

\subsection{Impact on rāga-mālikās}

We consider the impact of the algorithms on rāga-mālikās in Carnatic music. Rāga-mālikās, literally meaning garland of rāgas, are sequence of different rāgas. Generally rāga$s$ are so chosen as to be aesthetically pleasing and allied/ similar rāgas are usually avoided. The tonic frequency remains the same throughout the rendition. The challenge in rāga-mālikā rendition is in smoothly and seemlessly changing from one rāga to another - this necessitates precise depiction of $r \bar{g} g a$ characteristics within a short time duration. We analyse some such rāga-mālikās wherein each of the rendition is segmented into verses manually. ${ }^{4}$ These segments are tested against $P\left(s_{i} \mid r_{k}\right)$ that are estimated from the training set of Compmusic Carnatic rāga recognition dataset. Table 4 details rāga-mālikā compositions considered for the analysis. Table 4 tabulates the performance of various algorithms on the rāga-mālikā sections, followed by confusion matrices for 1-skip-3-gram and TDMS approaches in figure 14. We observe that though TDMS has a slight advantage in terms of rāga identification accuracy, the identification obtained using 1-skip-3-gram model maps better to musicologically similar rāgas. Thus, for durationconstrained test data, the proposed statistical models can be used to effectively reduce the search space (of rāgas) to grammatically similar rāgas.

\footnotetext{
${ }^{4}$ Generally, the first line of the pallavi part is repeated between two verses. We have ignored this repetitive line as we found this portion to be around $10 \mathrm{~s}$ duration and very unlikely to contribute to any meaningful statistics for evaluation.
}

Table 4. (a) Details of rāga-mālikā renditions considered for analysis. (b) Performance of the algorithms for the considered rāga-mālikā renditions.

\begin{tabular}{lc} 
(a) & \\
\hline No. of rāga-mālikās (unique, artists) & $5(3,5)$ \\
No. of $r \bar{a} g a$ segments & 25 \\
Average duration of a rāga segment & $72.4 \mathrm{~s}$ \\
\hline (b) & \\
\hline Algorithm & No. of correctly identified segments \\
3-gram & 4 \\
1-skip-3-gram & 14 \\
2-skip-3-gram & 8 \\
TDMS & 15 \\
\hline
\end{tabular}

\section{Visualization in embedded space}

The clusters of $P\left(r_{k} \mid d_{j}\right)$ are in the high-dimensional space $\left(\mathbb{R}^{R \times 1}\right)$. In order to visualize them, we embed them in a low-dimensional space. Earlier, we have depicted the formation of clusters using t-SNE plots [41].

\section{$6.1 t-S N E$}

t-SNE is a stochastic dimensionality reduction technique. The low-dimensional embedding preserves local structure to enable visualization of structures in high-dimensional large datasets.

The t-SNE technique matches the probability functions of data points in high dimensions and maps them to lowdimensional space. The similarity between points in lowdimensional space is modelled using Student- $t$ distribution, while those in high-dimensional space is modelled using a Gaussian distance measure. The cluster size and cluster distances are dependent on the perplexity (which is indicative of number of neighbours), a user-defined parameter of the algorithm [41]. Figure 15 shows t-SNE embedding of $P\left(r_{k} \mid d_{j}\right)$ for PLSA-based approach using skip-gram dictionaries. Figure 16 depicts t-SNE embedding of the melodic surface of TDMS obtained using two different bin resolutions.

\subsection{Observations from embedded space}

From the embedded space plots, which help in visualizing clusters, we observe that some clusters are closer than the others. We now make observations of the cluster closeness in terms of musicological similarities.

In the t-SNE plots of figure 15 corresponding to Carnatic dataset, eight major regions have been identified and highlighted with alphabetical markers (A-H). These regions correspond to ragas Harikāmbhōji, Karaharapriya, Thōdi, Kalyāni, Shanmukhapriya, Shankarābharana, 


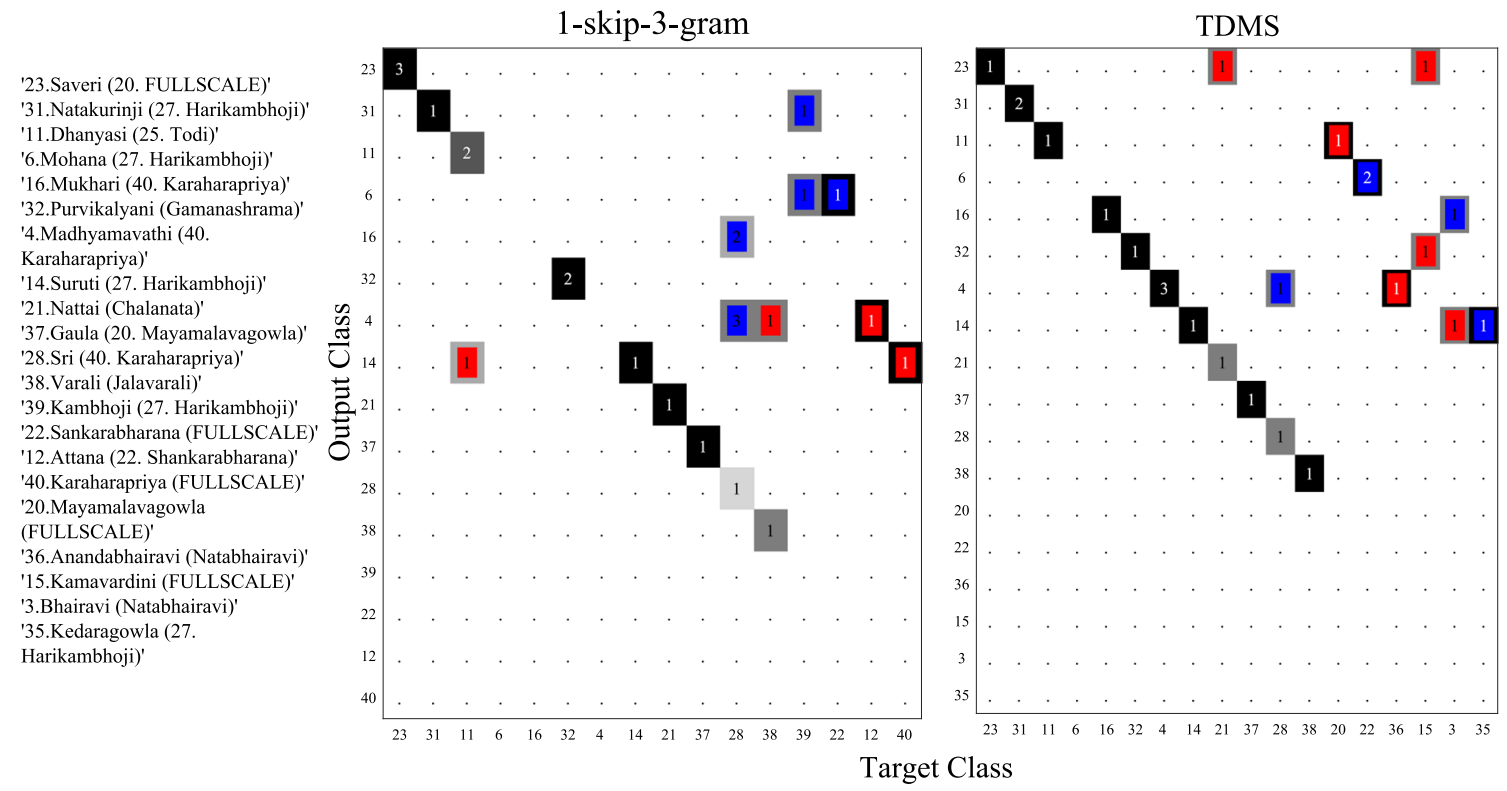

Figure 14. Confusion matrices for rāga-mālikā renditions using 1-skip-3-gram and TDMS models. Blue squares represent musicologically similar rāgas, while red squares highlight rāgas confused with dissimilar rāgas.

Māyamālavagowla and Kāmavardhini. These are full scale ragas. We note that allied ragas such as Thōdi and Dha$n y \bar{a} s i$ are close by. Similarly, ragas which are said to be Janya ragas (or non-full scale ragas) of Harikāmbhoji such as Khāmas, Kāmbhoji, Mōhana, Sahana and Surutti are found in close proximity. Other musicologically similar ragas found in close proximity are (i) Mayamālavagowla and Sāvēri, (ii) Shankarābharana, Bilahari, Bēgada and Behāg, (iii) Karaharapriya, Mukhāri, Sriranjani, Rītigowla, Husseni, Kānada, Sri, and (iv) Varāli and Kāmavardhini. However, we also observe some non-conformance to the Janaka-janya grouping such as (i) Behāg, Devagāndhāri, Yadukulakāmbhōji amongst Harikāmbhōji and Shankarābharana groups (groups A and F) owing to the usage of both $\mathrm{Ni}_{2}$ and $\mathrm{Ni}_{3}$ (Behāg being closer to Kalyāni can be ascribed to presence of $M a_{2}$ ), (ii) Bhairavi in Karaharapriya group - Because of its strong usage of $D a_{1}$, it is closer to Māyamalavagowla, but the rest of the notes/phrases are closer to Karaharapriya raga, (iii) Atāna not close to Shankaräbharana group - because of strong presence of $\mathrm{Ni}_{2}$ and (iv) Mōhana lies between Har$i k \bar{a} m b h \overline{o j i}$ and Shankarābharana clusters but closer to Shankarābharana cluster depending on the usage of phrases. We have also observed that the grouping does not reflect composer-specific RNP. The data-driven grouping obtained is interesting as it can partially explain some of the aspects and possible reasoning behind different and/or debatable viewpoints of Janaka-Janya grouping.

We see clusters of Khamaj, Goud Malhār of Khamaj Thāt (Group A), Basant and Puriya Dhanashrē belonging to Pūrvi Thāt (cf. http://www.itcsra.org/) (Group C), Yaman Kalyān and Kēdar of Kalyan Thāt (Group B) and Thōdi, Madhuvanthi and Bilasakhani Thōdi of Thōdi Thāt (Group
D). The ragas Bhoop and Dēsh are well separated with former being close to members of Kalyān Thāt (such as Yaman Kalyān) and latter being close to Khamaj Thāt members (such as Goud Malhār and Khamaj). However, we also observe for Hindustani dataset, not all raga-Thāt relations lie adjacent to other members of a Thāt - irregularities can be seen such as Shuddh Sārang not being close to Kāfi Thāt ragas (due to the presence of $\mathrm{Ma}_{2}$ and $\mathrm{Ni}_{3}$ and absence of $\mathrm{Ni}_{2}$ ), Mārwa and Lalit of Mārwa Thāt are not adjacent clusters owing to entirely different set of notes used.

Figure 16 shows t-SNE plots obtained from the TDMS technique to visualize the clusters with 2 different bin resolutions. We observe that the embeddings obtained with bin resolution of 10 cents (as proposed in the original work) do not reflect rāga-specific clustering of renditions, whereas clusters of renditions of same rāgas are seen with bin resolution of 100 cents. Considering the 100 cents bin resolution, we observe that the clusters do not reflect the musicological similarities across rāgas. ${ }^{5}$ This is because (i) TDMS can be viewed as a delayed QRP quantization technique, where the delay and quantization levels are empirically chosen and need not reflect successive or valid notes and (ii) TDMS is a delayed bi-gram [36] and bi-grams may not capture all the grammatical structure.

Similarity (or lack of) between the considered rāgas (in both Hindustani and Carnatic dataset) has been independently discussed with and corroborated by three musicologists. Thus, given a rendition we have explored the

\footnotetext{
${ }^{5}$ For e.g., from figure 16, we observe rāga Mōhana being depicted as close to rāga Dhanyāsi and rāga Kāmavardhini, which does not reflect musicological similarities.
} 
possibility of using the probability of a latent variable to construct a low-dimensional embedding, which reflects the musicological similarities in terms of rāga-renditions.
Thus, from the proposed algorithm, distances between renditions can be measured, musicological similarities between renditions can be measured and visualized. In

(I)
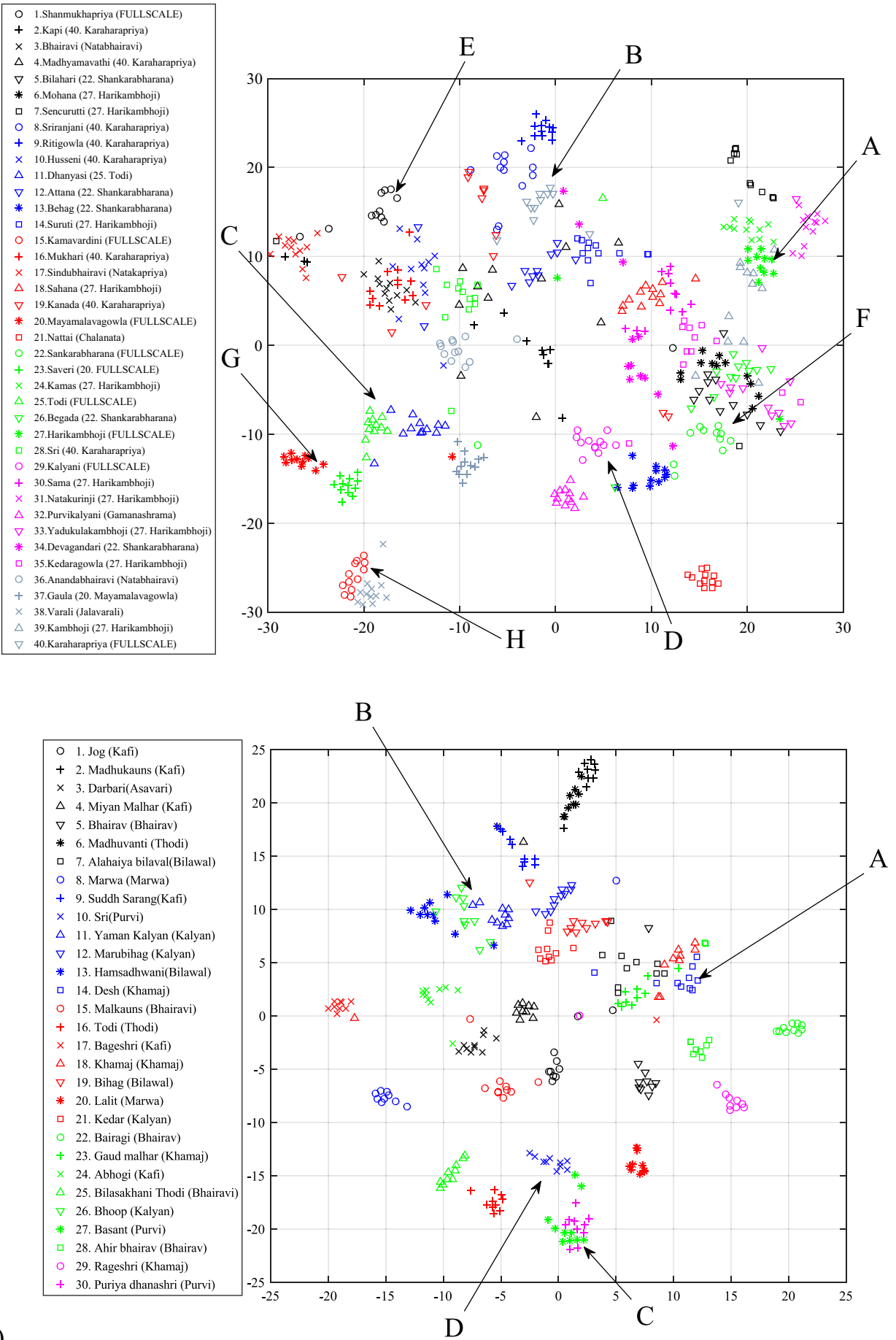

(II)

Figure 15. t-SNE embedding depicting low dimension representation of $P\left(r_{k} \mid d_{j}\right)$ obtained from $M$-skip- $N$-gram model for (I) Carnatic dataset with $(M=1, N=3)$ and (II) Hindustani dataset with $(M=1, N=4)$. For each rāga, the corresponding Melakarta/Thāt is mentioned in the brackets in the legend. Important regions are highlighted with the help of alphabetical markers (A-H). 

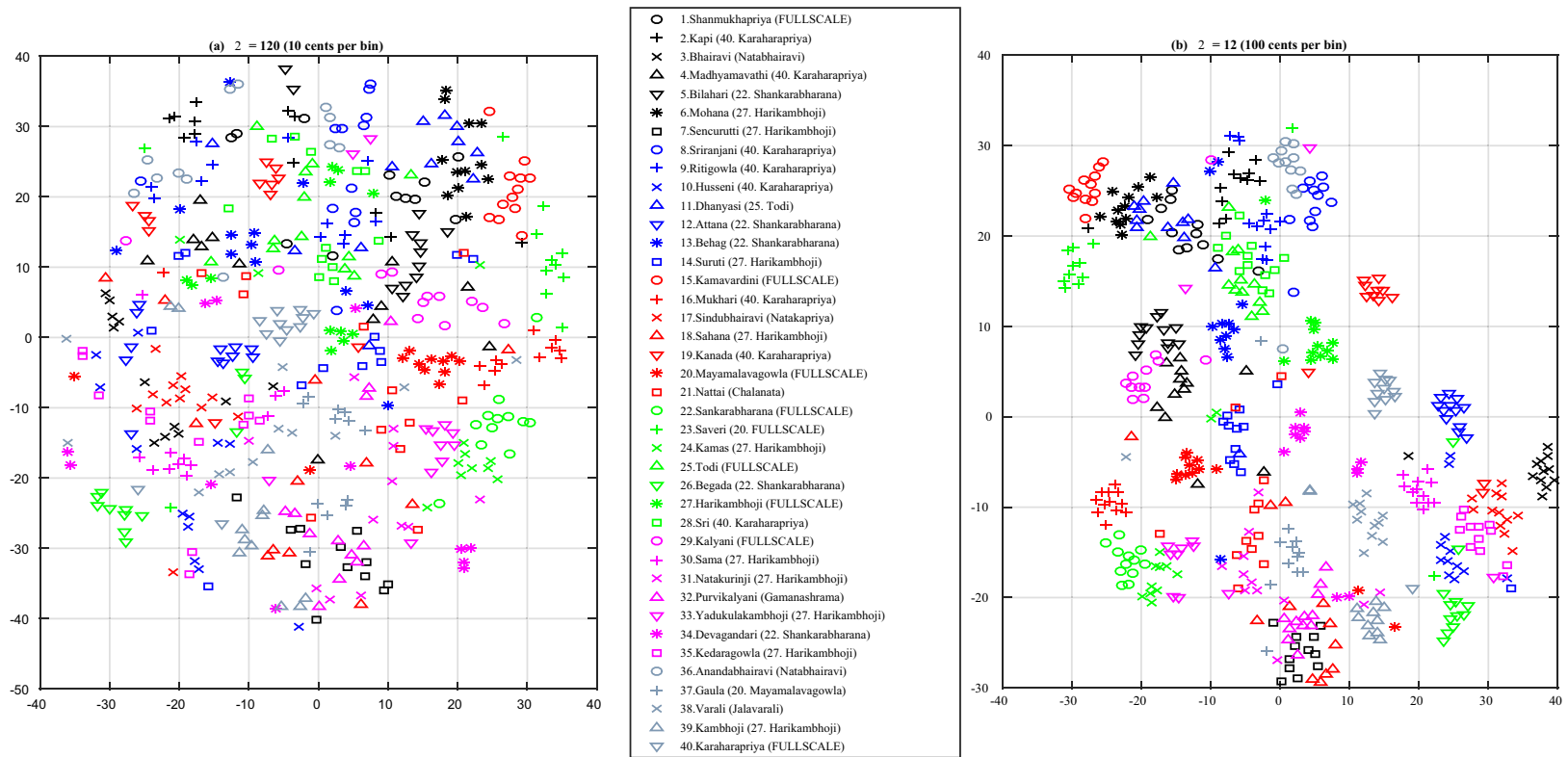

(II)
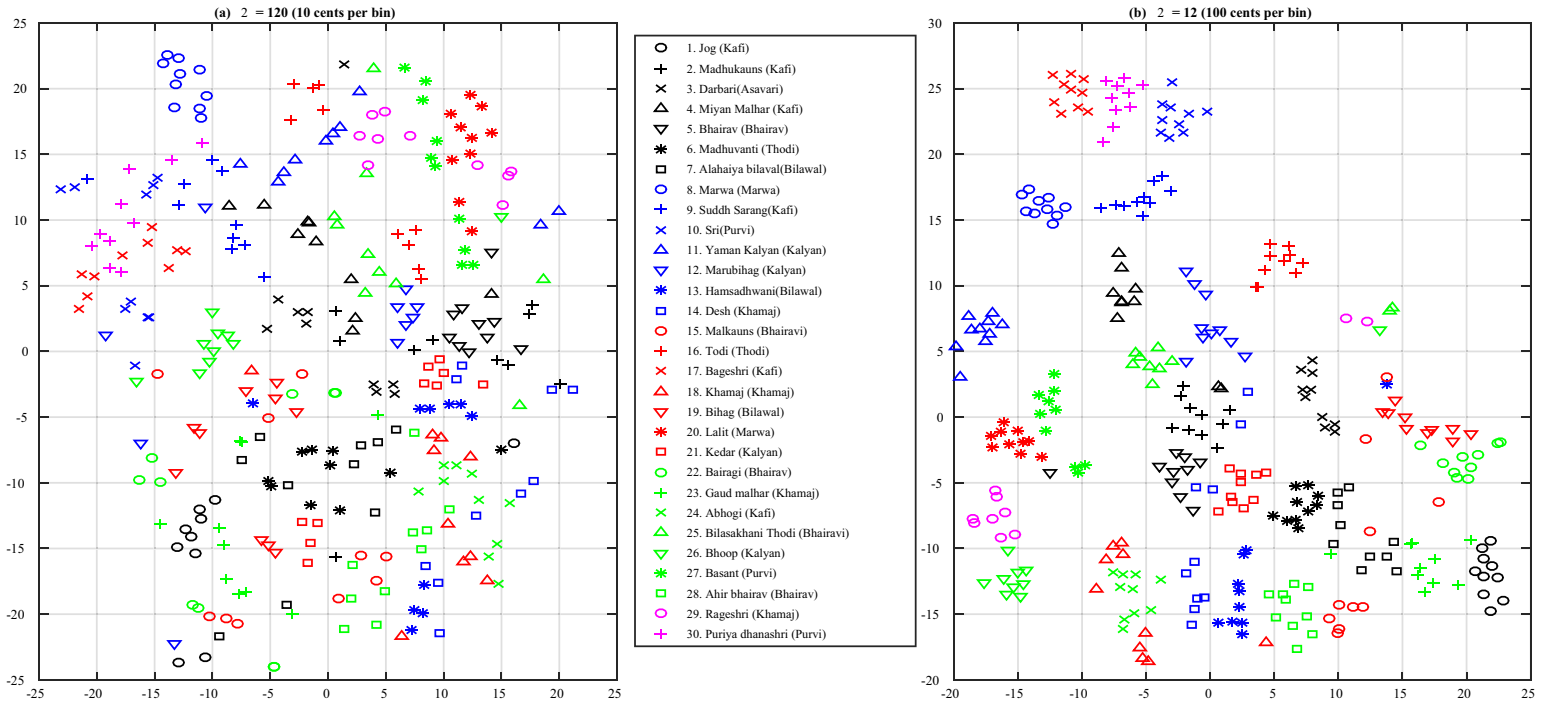

Figure 16. t-SNE embedding depicting low-dimension representation of TDMS technique for (I) Carnatic dataset and (II) Hindustani dataset. The left columns depict 10 cents per bin resolution and the right columns depict 100 cents per bin resolution.

addition, similar or allied rägas can be discovered using this approach.

\section{Conclusions}

We have proposed a framework for analysing melodic similarities by discretizing the contour. To evaluate the same, we have analysed $\sim 120 \mathrm{~h}$ each of Hindustani and Carnatic datasets and obtained descriptive note-level transcriptions. RNPs are formed from QCP and QRP subsequences and stochastic models of the same are obtained for each rendition in both Hindustani and Carnatic datasets.
We have observed that note transcriptions and stochastic models obtained with QCP quantization scheme is more effective for discovering $r a \bar{g} a$-similar clusters among renditions. They are shown to form a good feature representation for an IAM rendition. For rāga similarity analysis, we have proposed a model with latent variable for a $r \bar{a} g a$, akin to topic modelling in NLP. We have compared the performance of the proposed model to that of three other algorithms. We have seen that the proposed model is robust to duration of the rendition. In addition, the experiments on the datasets also assert that duration of rendition plays an important role particularly for Hindustani music form. We have used a data-driven approach to show that renditions of 
musicologically similar rāgas cluster in a latent space, thus proffering an approach for assessing musicological similarity. The rendition similarities are also visualized in a low-dimensional embedded space. Clustering and identification tasks are found to perform well with $M$-skip- $N$-gram dictionaries.

\section{Acknowledgements}

We thank Prof. Hema Murthy for her valuable guidance, inputs and comments in shaping the manuscript. We also thank Ch. Srikanth Raj for timely inputs for betterment of manuscript.

\section{References}

[1] Vijayakrishnan K G 2007 The grammar of Carnatic music, vol. 8. Berlin: Walter de Gruyter, Chapter 3

[2] Rajgopal Padma 1997 The study of Pundarika Vittala's treatises with reference to the systems of Raga classification in post-Ratnakara period. $\mathrm{PhD}$ Thesis, University of Baroda, Vadodara

[3] Ganguly O C 1947 Ragas and raginis. In: Nalanda books on ancient art, pp. 39-100

[4] Kaufmann W 1965 Rasa, rāga-mālā and performance times in north Indian rāgas. Ethnomusicology 9(3): 272-291

[5] Bhatkhande V N and Garga L 1990 Hindustani sangeet paddhati: bhatkhande kramik pustak. Sangeet Karyalaya, Hathras, Uttar Pradesh, India, pp. 17-30

[6] Sambamoorthy P 1998 South Indian music. Numbers 1-6. Indian Music Publishing House, Royapettah, Chennai, India

[7] Gulati S, Serrà J, Ishwar V, cSentürk S, Serra X 2016 Phrasebased rāga recognition using vector space modeling. In: Proceedings of the IEEE International Conference on Acoustics, Speech, and Signal Processing (ICASSP), pp. 66-70

[8] Dutta S, Krishnaraj Sekhar P V and Murthy H A 2015 Raga verification in Carnatic music using longest common segment set. In: Proceedings of the International Society on Music Information Retrieval Conference (ISMIR), pp. 605-611

[9] Gulati S, Serra J, Ishwar V and Serra X 2014 Mining melodic patterns in large audio collections of Indian Art Music. In: Proceedings of the IEEE International Conference on Signal-Image Technology and Internet-Based Systems (SITIS), pp. 264-271

[10] Padmasundari G and Murthy H A 2017 Raga identification using locality sensitive hashing. In: Proceedings of the National Conference on Communications (NCC), pp. 1-6

[11] Ishwar V, Dutta S, Bellur A and Murthy H A 2013 Motif spotting in an Alapana in Carnatic Music. In: Proceedings of the International Society on Music Information Retrieval Conference (ISMIR), pp. 499-504

[12] Dutta S and Murthy H A 2014 Discovering typical motifs of a raga from one-liners of songs in Carnatic music. In: Proceedings of the International Society on Music Information Retrieval Conference (ISMIR), pp. 397-402
[13] Dannenberg R B and Hu N 2003 Pattern discovery techniques for music audio. J. New Music Res. 32:153-163

[14] Janssen B, Bas De Haas W, Volk A and Van Kranenburg P 2013 Finding repeated patterns in music: state of knowledge, challenges, perspectives. In: Proceedings of the International Symposium on Computer Music Modeling and Retrieval (CMMR). Springer, Marseille, France, pp. 277-297

[15] Şentürk S, Koduri G K and Serra X 2016 A score-informed computational description of svaras using a statistical model. In: Proceedings of the Conference on Sound and Music Computing (SMC), Germany, pp. 427-433

[16] Koduri G K 2016 Towards a multimodal knowledge base for Indian Art Music: a case study with melodic intonation. $\mathrm{PhD}$ Thesis, Universitat Pompeu Fabra, Barcelona

[17] Ranjani H G, Paramashivan D and Sreenivas T V 2017 Quantized melodic contours in Indian Art Music perception: application to transcription. In: Proceedings of the International Society on Music Information Retrieval Conference (ISMIR), pp. 174-180

[18] McAdams S 1989 Psychological constraints on form-bearing dimensions in music. Contemp. Music Rev. 4: 181-198

[19] Caragea C, Silvescu A and Mitra P 2011 Protein sequence classification using feature hashing. In: Proceedings of the IEEE International Conference on Bioinformatics and Biomedicine, pp. 538-543

[20] La Rosa M, Fiannaca A, Rizzo R and Urso A 2015 Probabilistic topic modeling for the analysis and classification of genomic sequences. BMC Bioinformatics 16: S2 (1-9)

[21] Airoldi E M, Blei D M, Fienberg S E and Xing E P 2008 Mixed membership stochastic blockmodels. J. Mach. Learn. Res. 9: 1981-2014

[22] Cambria E and White B 2014 Jumping NLP curves: a review of natural language processing research [review article]. IEEE Comput. Intell. Mag. 9: 48-57

[23] Griffiths T L, Steyvers M and Tenenbaum J B 2007 Topics in semantic representation. Psychol. Rev. 114: 211-244

[24] Smaragdis P, Raj B and Shashanka M 2007 Supervised and semi-supervised separation of sounds from single-channel mixtures. In: Proceedings of the International Conference on Independent Component Analysis and Signal Separation. Springer, pp. 414-421

[25] Shashanka M, Raj B and Smaragdis P 2008 Probabilistic latent variable models as non-negative factorizations. Comput. Intell. Neurosci.https://doi.org/10.1155/2008/947438

[26] Gulati S, Serrà J, Ganguli K K, Şentürk S and Serra X 2016 Time-delayed melody surfaces for rāga recognition. In: Proceedings of the International Society on Music Information Retrieval Conference (ISMIR), pp. 751-757

[27] Ranjani H G, Arthi S and Sreenivas T V 2011 Carnatic music analysis: Shadja, Swara identification and Raga verification in Alapana using stochastic models. In: Proceedings of the IEEE Workshop on Application of Signal Processing to Audio and Acoustics (WASPAA), pp. 29-32

[28] Guthrie D, Allison B, Liu W, Guthrie L and Wilks Y 2006 A closer look at skip-gram modelling. In: Proceedings of the International Conference on Language Resources and Evaluation (LREC), pp. 1-4

[29] Deligne S and Bimbot F 1995 Language modeling by variable length sequences: theoretical formulation and evaluation of multigrams. In: Proceedings of the IEEE International 
Conference on Acoustics, Speech and Signal Processing (ICASSP), vol. 1, pp. 169-172

[30] Bogdanov D, Wack N, Gómez E, Gulati S, Herrera P, Mayor O, Roma G, Salamon J, Zapata J R and Serra X 2013 Essentia: an audio analysis library for music information retrieval. In: Proceedings of the International Society on Music Information Retrieval Conference (ISMIR), pp. 493-498

[31] Blei D M 2012 Probabilistic topic models. Commun. ACM 55: 77-84

[32] Cohn D A and Hofmann T 2001 The missing link-a probabilistic model of document content and hypertext connectivity. In: Advances in neural information processing systems, pp. 430-436

[33] Manning C D, Raghavan P and Schütze H 2008 Introduction to information retrieval. New York: Cambridge University Press

[34] Vidwans A and Rao P 2012 Identifying Indian classical music styles using melodic contours. In: Proceedings of the Conference on Frontiers of Research in Speech and Music (FRSM)
[35] Viraraghavan V S, Aravind R and Murthy H A 2017 A statistical analysis of gamakas in carnatic music. In: Proceedings of the International Society on Music Information Retrieval Conference (ISMIR), pp. 243-249

[36] Ranjani H G and Sreenivas T V 2017 Raga identification using repetitive note patterns from prescriptive notations of Carnatic music. arXiv:1711.11357

[37] van der Maaten L and Hinton G 2008 Visualizing data using t-SNE. J. Mach. Learn. Res. 9: 2579-2605

[38] Akaike H 1974 A new look at the statistical model identification. IEEE Trans. Autom. Control 19: 716-723

[39] Chordia P and Şentürk S 2013 Joint recognition of raag and tonic in north Indian music. Comput. Music J. 37: 82-98

[40] Mikolov T, Kopecky J, Burget L, Glembek O et al 2009 Neural network based language models for highly inflective languages. In: Proceedings of the IEEE International Conference on Acoustics, Speech and Signal Processing (ICASSP), pp. 4725-4728

[41] Wattenberg M, Vigas F and Johnson I 2016 How to use t-SNE effectively. http://distill.pub/2016/misread-tsne 